\title{
Generalizing the self-healing diffusion Monte Carlo approach to finite temperature: a path for the optimization of low-energy many-body bases
}

\author{
Fernando A. Reboredo and Jeongnim Kim \\ Materials Science and Technology Division, \\ Oak Ridge National Laboratory, Oak Ridge, TN 37831, USA
}

(Dated: February 11, 2018)

\begin{abstract}
A statistical method is derived for the calculation of thermodynamic properties of many-body systems at low temperatures. This method is based on the self-healing diffusion Monte Carlo method for complex functions [F. A. Reboredo J. Chem. Phys. 136, 204101 (2012)] and some ideas of the correlation function Monte Carlo approach [D. M. Ceperley and B. Bernu, J. Chem. Phys. 89, 6316 (1988)]. In order to allow the evolution in imaginary time to describe the density matrix, we remove the fixed-node restriction using complex antisymmetric guiding wave functions. In the process we obtain a parallel algorithm that optimizes a small subspace of the many-body Hilbert space to have maximum overlap with the subspace spanned by the lowest-energy eigenstates of a many-body Hamiltonian. We show in a model system that the partition function is progressively maximized within this subspace. We show that the subspace spanned by the small basis systematically converges towards the subspace spanned by the lowest energy eigenstates. Possible applications of this method to calculate the thermodynamic properties of many-body systems near the ground state are discussed. The resulting basis can be also used to accelerate the calculation of the ground or excited states with Quantum Monte Carlo.
\end{abstract}

PACS numbers: $02.70 . \mathrm{Ss}, 02.70 . \mathrm{Tt}$ 


\section{INTRODUCTION}

There is a significant interest in thermodynamical properties observed as $T \rightarrow 0$. Many physical phenomena that cover superconductivity, magnetic and structural transitions, chemical reactions etc. require an adequate treatment of thermal effects. These effects are crucial in systems where there is a large number of low-energy excitations within an energy window $1 / \beta=k_{B} T$ above the ground state. Electronic thermal effects are expected to be larger in metals and magnets than in insulators. $\frac{1}{-}$ In metals there is a significant number of excitations with vanishing energy. The magnetic excitations energies frequently go to zero in the long wave limit. A significant fraction of spectroscopic techniques probe the electronic or magnetic excitations near the ground state. The development of first-principles techniques to obtain excitations has historically received a significant theoretical attention..$^{2-4}$ Monte Carlo methods used to calculate excitation energies ${ }^{4}$ will be accelerated with basis that retain the physics at the relevant energies.

A first-principles finite-temperature description of many-body systems is also relevant to describe chemical reactions. $\frac{5}{-}$ Ionic dynamics are usually calculated within the Born-Oppenheimer approximation. This decouples the wave function of the "quantum" electrons from the wave function of the ions. Within this standard approximation, electrons are at zero temperature while the ions can move with kinetic energies that often exceed the electronic excitations. Even within the Born-Oppenheimer-ground-state approximation, the standard approach based on density func-

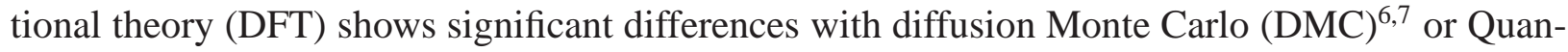
tum Chemistry benchmarks. At the transition saddle points, when some chemical bonds are broken and new ones are formed, the spacing of the corresponding electronic eigenenergies is minimum, or even zero at the conical intersections..$^{8}$ Electronic thermal effects are seldom included in manybody calculations.. .9 In order to routinely include thermal effects, significant improvements in the theory beyond the standard approach are required.

Most ab-initio calculations in the literature of condensed matter electronic structure are based in the ground state quantum Monte Carlo calculations of the homogeneous electron gas $\underline{10}$ which made possible the first approximations of DFT: $\stackrel{11,12}{ }$ DFT has been extended to finite temperature long ego $\stackrel{13-15}{-15}$ Fermi occupations of Kohn-Sham eigenstates and the addition of an entropy term have been shown ${ }^{15}$ to provide a variational density functional. However, even nowadays, the zero temperature approximation for the exchange-correlation potential is widely used. This approach has been long known to severely underestimate the critical Curie temperatures of magnetic sys- 
tems. $\underline{16}-\underline{18}$ Including temperature for magnetic systems is possible for cases where the magnetic excitations can be treated classically $\underline{19.20}$ and the electrons can be assumed to be in the ground state for constrained configurations of the spins. $\frac{21}{3}$ But an adequate description of the electronic entropy in the subspace that preserves the spin is still lacking. $\underline{\underline{22}}$ Finite temperatures benchmarks of a quality comparable to Ref. 10 are the key ingredients required to parametrize a finite temperature density functional. Without a reliable approximation, most work done under a DFT framework still uses a zero temperature approximation for the exchange correlation functional.

Accurate many-body calculations at high temperatures can be performed within the path integral Monte Carlo approach (PIMC) $\stackrel{23}{\underline{2}}$ Since the cost of PIMC diverges as $T \rightarrow 0$, it has been mainly used in the hot and dense regime, $\underline{\underline{24}}-\underline{26}$ with a temperature comparable to the interaction potential. An alternative approach that could start from the zero temperature limit would be desirable.

The most accurate techniques to describe a large number of electrons $\left(N_{e}>1000\right)$ at zero temperature are based in projection approaches. $\frac{10.27 .28}{1}$ One could potentially extend these methods to finite temperature, limiting the projector $e^{-\beta \hat{\mathcal{H}}}$ to finite $\beta$. Thermodynamical averages can be later obtained from derivatives of the Helmholtz free energy $F(\beta)=-1 / \beta \ln \left[\operatorname{tr}\left(e^{-\beta \hat{\mathcal{H}}}\right)\right]$, where $\hat{\mathcal{H}}$ is the many-body Hamiltonian operator and $\operatorname{tr}(X)$ the trace of $X$ over the complete many-body Hilbert space.

The standard diffusion Monte Carlo Method with importance sampling (DMC) ${ }^{10,29.30}$ constrains the sign or the phase of the wave function by imposing the nodes or the phase $\mathrm{3l}^{\underline{\underline{3}}}$ of a guiding wave function $\Psi_{T}(\mathbf{R})$, where the many-body coordinate $\mathbf{R}=\left\{\mathbf{r}_{1}, \mathbf{r}_{2}, \cdots, \mathbf{r}_{N_{e}}\right\}$ is the set of coordinates of $N_{e}$ electrons. These constraints while enforcing an anti-symmetric fermionic wave function introduce a variational error. The quality of the wave function and its nodes can be improved with several methods within a variational Monte Carlo (VMC) context, $\underline{29.32} \underline{\underline{38}}$ or at the DMC level $\stackrel{33,39-42}{-4 n}$ DMC, the energy of the ground state is exact if the exact nodes or phase are provided. $\frac{31,43}{2 m p r o v i n g ~ t h e ~ n o d e s ~ i s ~ c o m p u t a t i o n a l l y ~ i n t e n s i v e . ~ A v o i d i n g ~ t h i s ~ c o s t ~ i s ~ k e y ~ f o r ~ f i n i t e ~}$ temperature calculations.

In standard DMC calculations, $\hat{\mathcal{H}}$ is replaced by the fixed-node Hamiltonian $\hat{\mathcal{H}}_{F N}$ or the fixedphase Hamiltonian $\hat{\mathcal{H}}_{F P}$. The use of the fixed-node or fixed phase approximation can have undesired effects on the calculation of thermal effects. It has been found that many fermionic systems have a ground state with two nodal pockets. ${ }^{44}$ That is, if the ground state wave function is real the nodal surface separates the Hilbert space in only two pockets for positive and negative values 
respectively. It has been conjectured ${ }^{45}$ that this is a general property of fermionic ground states. In the fixed-node case, the excitations of $\hat{\mathcal{H}}_{F N}$ are forced to share the nodes of the ground state. To be orthogonal to the fixed-node ground state, the fixed-node excited states have to have at least an additional node. It is known, however, that in many systems there are several fermionic excited states near the ground state with also two nodal pockets. $\underline{\underline{46}}$ Accordingly, $\operatorname{tr}\left(e^{-\beta \hat{\mathcal{H}}_{F N}}\right)$ does not describe the low temperature physics. It is easy to see that the same happens in the fixed-phase case. Therefore, if one wishes to use a DMC-like algorithm to obtain thermodynamical properties, one must go beyond the usual fixed-node or fixed-phase approximations. For practical reasons, a parallel approach that can handle a large number of excitations near the ground state would also be beneficial.

In this paper, we restart the debate on how to calculate low temperature properties within a many-body ab-initio context taking into account recent theoretical developments. ${ }^{40,42,47}$ A method is derived that introduces temperature within an importance sampling procedure that shares most of the computational tools developed for projection MC approaches. The errors in the evolution operator $e^{-\beta \hat{\mathcal{H}}}$ resulting from the fixed-node restriction are eliminated by using complex linear combinations of eigenstates, which do not have nodes except at the electronic coincidental points (see Fig 1). Instead of optimizing a single many-body wave-function so it better describes the ground state of the system, a basis of several wave functions is optimized to maximize the overlap with the small subspace spanned by the lowest energy eigenstates of the many-body Hamiltonian. We show in a model system that the overlap of the optimized subspace with the lowest energy subspace calculated with a configuration interaction (CI) approach, increases systematically as the iterations increase and that the partition function is maximized.

The rest of the paper is organized as follows: In Section $\llbracket$ we describe the general formalism; some of the formulae developed in Ref. 42 for complex wave function is repeated here for completeness. Section $\amalg$ outlines the basic algorithm. In Section $I V$ we describe the results for a model calculation; and finally, in Section $\nabla$ we discuss the possible applications and summarize. This paper also has three appendices: $\mathrm{A}$ describes how to go beyond the locality and local-time approximations; $\mathrm{B}$ describes how to take advantage of the eigenstates when they are complex; Finally, $\mathrm{C}$ describes how to work with eigenstate pairs to minimize the variance of the weights of the walkers while keeping the wave function complex. 


\section{A LOW-ENERGY EXPANSION OF THE PARTITION FUNCTION}

This section extends the DMC approach ${ }^{10}$ for the calculation of the partition function of a many-body system. We first provide background material required to understand the rest of the paper. We generalize the upper bound property of the energy in DMC to an upper bound property of the free energy. We next give the general outline of our approach and describe how to avoid the fixed-node approximation in DMC. Finally, we describe the details: basic formulae and numerical approach.

\section{A. The upper bound property of the truncated Helmholtz free energy}

Thermal effects can be obtained by calculating all excitations within a thermal energy window above the ground state larger than $\Delta E \sim 1 / \beta=k_{B} T$ and then evaluating the density matrix ${ }^{48}$ as:

$$
\begin{aligned}
\hat{\rho}(\beta) & =e^{-\beta \hat{\mathcal{H}}} \\
& =\sum_{n}\left|\Psi_{n}\right\rangle e^{-\beta E_{n}}\left\langle\Psi_{n}\right|,
\end{aligned}
$$

where $E_{n}<E_{0}+\Delta E$ is the eigenvalue with eigenvector $\left|\Psi_{n}\right\rangle$ of $\hat{\mathcal{H}}$. In general $\hat{\mathcal{H}}$ is given by

$$
\hat{\mathcal{H}}=\sum_{j}^{N_{e}} \frac{\left(\nabla_{j}+\mathbf{A}_{j}\right)^{2}}{2}+\hat{V}(\mathbf{R})
$$

where $\mathbf{A}_{j}=\mathbf{A}\left(\mathbf{r}_{j}\right)$ is a vector potential at point $\mathbf{r}_{j}$ with magnetic field $\mathbf{B}\left(\mathbf{r}_{j}\right)=\nabla_{j} \times \mathbf{A}_{j}$, and $\hat{V}(\mathbf{R})$ includes the electron-electron interaction, the interactions of the electrons spins with the magnetic field and any external potential, local or non-local.

In a closed system that can exchange energy with a bath or reservoir (canonical ensemble) all thermodynamical averages can be obtained using the density matrix. The trace of the density matrix $Z(\beta)=\operatorname{tr}[\rho(\beta)]$ is the partition function, whereas $F(\beta)=-1 / \beta \ln [Z(\beta)]$ is the Helmholtz free energy.

In general, $\hat{\mathcal{H}}$ has an infinite number of eigenvectors $\left|\Psi_{n}\right\rangle$ that can be ordered with increasing eigenenergy $E_{n}$. If $\beta\left(E_{n}-E_{0}\right) \gg 1$, the contribution to $Z(\beta)$ of the eigenstate $\left|\Psi_{n}\right\rangle$ becomes negligible. Therefore, a usual approximation is to truncate the trace to a finite matrix with a finite number of eigenstates $M_{S}$.

In what follows we defined $\operatorname{tr}()$ as the trace of a truncated square matrix with size $M_{S}$. We also relate $Z(\beta)$ and $F(\beta)$ to that truncated trace. Since $e^{-\beta \hat{\mathcal{H}}}$ is positive definite, for a given basis, 
$Z(\beta)$ increases and $F(\beta)$ decreases as $M_{S}$ increases.

The trace of any linear operator is invariant for linear transformations of the form $\hat{B} e^{-\beta \hat{\mathcal{H}}} \hat{B}^{-1}$ with $\hat{B} \hat{B}^{-1}=1$. Thus, in principle, one does not need to obtain the eigenstates of $e^{-\beta \hat{\mathcal{H}}}$ or equivalently $\hat{\mathcal{H}}$ to calculate the free energy. Any linearly independent basis that spans the same subspace can be used to obtain $Z(\beta)$. Thermodynamical properties only require us to evaluate $Z(\beta)$ in a linearly independent basis $\left\{\left|\chi_{m}^{S}\right\rangle\right\}$. However, if statistical methods are used, then each element contributing to the truncated trace also increases the statistical error bar. Therefore, it is computationally more efficient to use the most compact basis, with minimum $M_{S}$, that retains the low-energy properties.

Any eigenstate $\left|\Psi_{n}\right\rangle$ can be written in a complete basis $\left|\chi_{n}^{S}\right\rangle$ as

$$
\left|\Psi_{n}\right\rangle=e^{-\hat{J}}\left[\sum_{m=0}^{M_{S}-1} \lambda_{n}^{m}\left|\chi_{m}^{S}\right\rangle+\sum_{m=M_{S}}^{N_{B} \rightarrow \infty} \lambda_{n}^{m}\left|\chi_{m}^{S}\right\rangle\right],
$$

where $\left\langle\mathbf{R}\left|e^{-\hat{J}}\right| \mathbf{R}\right\rangle=e^{-J(\mathbf{R})}$ is a Jastrow factor that introduces adequate cusp conditions ${ }^{49}$ and are $\left\langle\mathbf{R} \mid \chi_{m}^{S}\right\rangle=\chi_{m}^{S}(\mathbf{R})$ linear combinations of an infinite orthogonal set $\{\langle\mathbf{R} \mid n\rangle\}$ [e.g. Slater determinants, or Pfaffians $\stackrel{50}{ }$, or symmetry constrained functions (SCF), etc]. In practice we restrict the Hilbert space to a finite number $N_{B}$. We denote the subspace spanned by $N_{B} \gg M_{S}$ functions $|m\rangle$ as the big subspace. The big subspace has to be large enough to describe the low temperature physics of the complete Hilbert space, which is in general infinite. We define the small subspace as the subspace spanned by the first $M_{S}$ basis functions $\left|\chi_{n}^{S}\right\rangle$.

Within the small basis $\left\{\left|\chi_{m}^{S}\right\rangle\right\}$, the free energy will be minimum if all $\left|\Psi_{n<M_{S}}\right\rangle$ can be spanned in the small basis, namely, $\lambda_{n \geq M_{S}}^{m}=\lambda_{n}^{m \geq M_{S}}=0$. Errors in the small basis will result in higher values of the free energy. Thus the free energy in the truncated basis is an upper bound to the true Helmholtz free energy. Optimization of the Helmholtz free energy in the small basis is analogous to the variational principle of the ground state. Likewise, the partition function in the truncated basis is a lower bound of the exact partition function. Improved bounds may be obtained with a basis that better describes the lower energy eigenstates. $M_{S}$ has to be large enough to include all the relevant physics for a given temperature.

Most of the optimization methods in the QMC literature focus, on optimizing the eigenstates. Several methods have been proposed to obtain low-energy excited states within the linear method Monte Carlo $\div .51$ (LMMC) or diffusion Monte Carlo. $\stackrel{39.47}{.4}$ However, since the eigenstates are sometimes difficult calculate, and we only need an average, we argue that one might save computational time by optimizing the many-body basis first as in the correlation function diffusion Monte Carlo 
(CFDMC $)^{39}$ method. Optimizing the basis directly could be more practical than obtaining accurate eigenstates energies, if the number of excitations near the ground state is large (e. g. typically the case in metallic or magnetic systems).

\section{B. Guiding ideas of the finite temperature SHDMC method and definitions}

Instead of performing the usual projection for infinite imaginary time of a single trial wavefunction, we run DMC for multiple guiding wave-functions (forming a linearly independent basis) for finite imaginary time, which is equivalent to finite temperature. Instead of using a single real guiding function with nodes, we use a set of complex antisymmetric guiding functions without nodes. Therefore, the Hamiltonian $\hat{\mathcal{H}}$ is not altered at the nodes as in the standard importance sampling DMC approach $\underline{10}$ with the fixed-node approximation. ${ }^{43}$ As explained in the introduction, extending DMC to finite temperatures requires to go beyond those fixed schemes. Complex-valued antisymmetric wave functions, that do not have nodal pockets, can be constructed as a linear combination of two real wave function with different nodes (see Fig. 11). We go beyond the standard fixed-phase approximation and the local-time approximation. ${ }^{42}$ As in the SHDMC method for complex wave functions,,$\frac{42}{-}$ the infamous sign problem is avoided with complex antisymmetric guiding functions. The result is acurate as long as enough statistical information is collected.

SHDMC ${ }^{40,42,47}$ systematically improves a trial wave function by maximizing the overlap with the wave function propagated in imaginary time in DMC. Here instead of maximizing the overlap of a single wave function we will maximize the overlap with the basis.

Following the ideas of the SHDMC method, we use a recursive approach. In every iteration $\ell$, importance sampling $\mathrm{DMC}^{10}$ is performed and statistical data of the evolution in $\beta$ of a set of $M_{S}$ guiding wave functions is projected on the many-body bases $\left\{\left|\chi_{n}^{S, \ell}\right\rangle\right\}$ and $\{|n\rangle\}$. The statistical data is used to improve the small basis $\left\{\left|\chi_{n}^{S, \ell+1}\right\rangle\right\}$ and the guiding functions for the next iteration In what follows, we will omit the iteration index $\ell$ in the notation for clarity, when the basis is not changed. The small basis $\left\{\left|\chi_{n}^{S}\right\rangle\right\}$ is orthonormal: $\left\langle\chi_{n}^{S} \mid \chi_{n}^{S}\right\rangle=\delta_{n, m}$.

For numerical efficiency, depending on the problem, we choose guiding functions related to the eigenstates of $\hat{\mathcal{U}}=e^{\hat{J}} e^{\hat{\mathcal{H}}} e^{-\hat{J}}$. (i) $\left\{\left|\chi_{n}^{U}\right\rangle\right\}$ is formed by the Slater expansions of the eigenstates in the small basis. (ii) $\left\{\left|\chi_{n}^{V}\right\rangle\right\}$ is formed by the Slater expansion of linear combinations of eigenstates pairs. 
We construct wave functions of the form

$$
\Psi_{n}^{T}(\mathbf{R})=e^{-J(\mathbf{R})} \chi_{n}^{X}(\mathbf{R})
$$

where the super index $X$ refers to either $S, U$, or $V$, depending on the case. To simplify the notation, we omit $X$ in $\Psi_{n}^{T}(\mathbf{R})$.

We assume the Jastrow factor operator $e^{-\hat{J}}$ to be diagonal in the many-body configuration

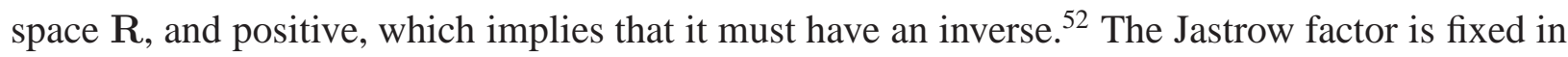
SHDMC but can be optimized variationaly so that the free energy of the system is minimized.

In contrast with the CFDMC ${ }^{39}$ and the released phase ${ }^{33}$ methods, we use anti-symmetric guiding functions, which are improved recursively with a maximum overlap criterion. Since the exponential growth of the bosonic ground state is prevented by the guiding functions, the free energy obtained is an upper bound. This approach is different to the correlated linear method ${ }^{4}$ because the wave function is optimized at the DMC level and we use anti-symmetic guiding functions. In variance with the original SHDMC approach for excited states $\frac{47}{3}$ multiple wave functions are propagated in parallel. A serial orthogonalization step in the original SHDMC method for excited states $^{42.47}$ is postponed in this new approach until DMC has been run for the all basis functions. ${ }^{53}$

\section{Working with complex guiding wave functions to avoid the fixed-node approximation}

While complex guiding wave functions allow us to avoid the fixed-node approximation, they introduce additional complications $\stackrel{42}{ }$ that are discussed here. Once these complications are dealt with, the sign problem is avoided as in the fixed-node. As in standard SHDMC the result is accurate as long as enough statistics is obtained.

Following Refs. 31 and $42, \Psi_{n}^{T}(\mathbf{R})=\left\langle\mathbf{R} \mid \Psi_{n}^{T}\right\rangle$ can be written as an explicit product of a complex phase and an amplitude $\Psi_{n}^{T}(\mathbf{R})=\Phi_{n}^{T}(\mathbf{R}) e^{\mathbf{i} \phi_{n}(\mathbf{R})}$.

The expressions

$$
\begin{aligned}
\Phi_{n}^{T}(\mathbf{R}) & =\sqrt{\Psi_{n}^{T}(\mathbf{R}) \Psi_{n}^{T *}(\mathbf{R})}, \text { and } \\
\phi_{n}(\mathbf{R}) & =\ln \left[\Psi_{n}^{T}(\mathbf{R}) / \Psi_{n}^{T *}(\mathbf{R})\right] /(2 i)+\pi m
\end{aligned}
$$

allow the computation of all the gradients and Laplacians in terms of those of an arbitrary complex function $\chi_{n}^{X}(\mathbf{R})$ and $e^{-J(\mathbf{R})}$.

In Eq. (6) $m$ is an arbitrary integer that changes the Riemann branch of the natural logarithm ln of a complex number. $m$ only contributes to the gradient or Laplacian at the Reimann cuts. $\underline{54}$ 


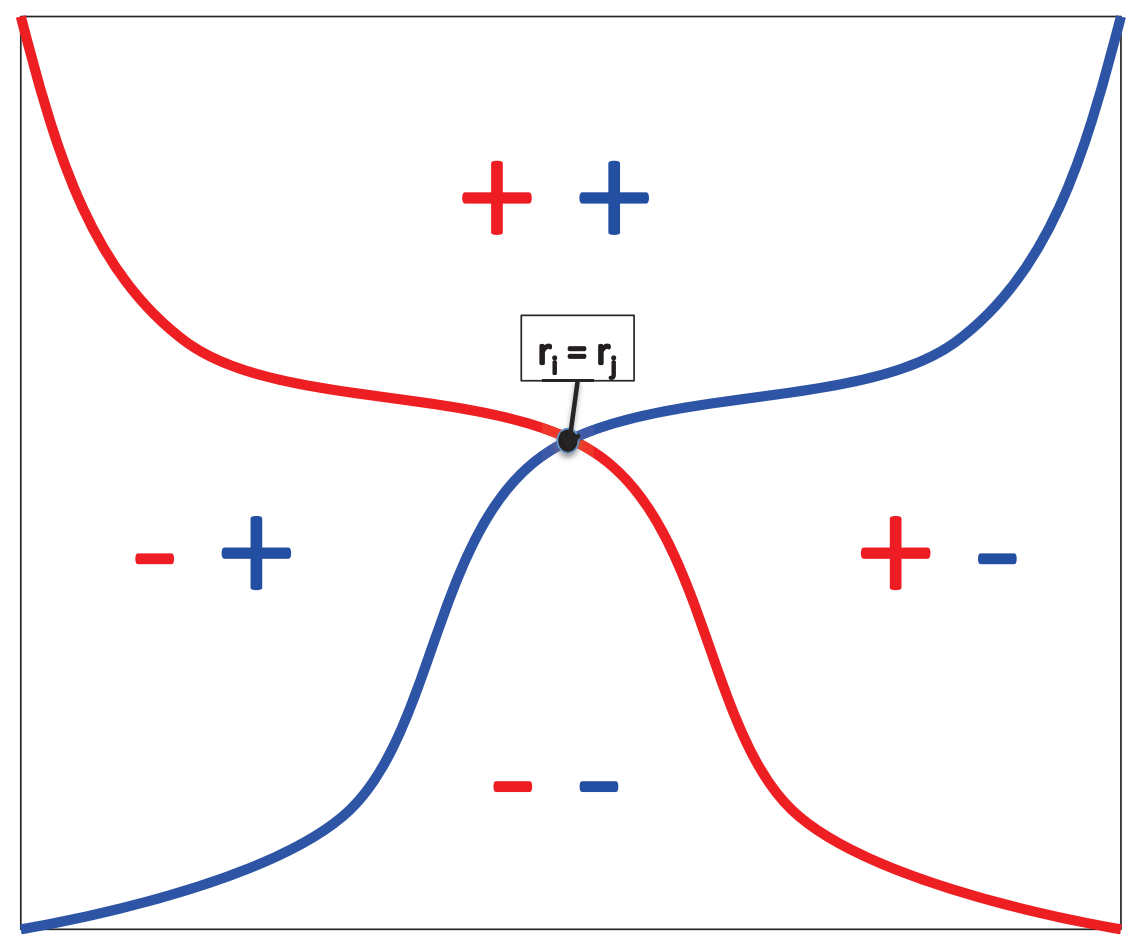

FIG. 1: (Color online) A schematic representation of the nodes of the real and imaginary parts of a complex antisymmetric function. This type of complex functions must be zero only at the coincidental points. Though real antisymmetric functions must have nodal pockets a complex linear combination must not. Note also that two different antisymmetric real wave functions approaching different eigenstates will have different nodes and produce different fixed-node Hamiltonians.

Since the position of the Reimann cuts is an arbitrary mathematical convention, their contribution to gradients and Laplacians is unphysical and ignored.

The dependence in $\beta$ of $e^{-\beta \hat{\mathcal{H}}} \Psi_{n}^{T}(\mathbf{R})$ is given by

$$
\begin{aligned}
\Psi_{n}^{T}(\mathbf{R}, \beta) & =e^{-\beta \hat{\mathcal{H}}} \Psi_{n}^{T}(\mathbf{R}) \\
& =e^{-\beta \hat{\mathcal{H}}}\left[\Phi_{n}^{T}(\mathbf{R}) e^{\mathbf{i} \phi_{n}(\mathbf{R})}\right] \\
& =\Phi_{n}^{T}(\mathbf{R}, \beta) e^{\mathbf{i} \phi_{n}(\mathbf{R})}
\end{aligned}
$$

Equation (9) includes by definition all the temperature dependence in 
$\Phi_{n}^{T}(\mathbf{R}, \beta)=\Psi_{n}^{T}(\mathbf{R}, \beta) / e^{\mathbf{i} \phi_{n}(\mathbf{R})}$, denoted as free-amplitude, since it can be complex, $\underline{42}$ with $\Phi_{n}^{T}(\mathbf{R}, 0)=\Phi_{n}^{T}(\mathbf{R})$. The phase $\phi_{n}(\mathbf{R})$ remains fixed in the interval $[0, \beta)$ as in Ref. 31

Following Ref. 42 we define the quantity

$$
f_{n}(\mathbf{R}, \beta)=\Psi_{n}^{T *}(\mathbf{R}) \Psi_{n}^{T}(\mathbf{R}, \beta) e^{\beta E_{n}^{X}},
$$

where $E_{n}^{X}$ is a reference energy adjusted numerically to satisfy the condition $\left\langle\chi_{n}^{X}\left|e^{\hat{J}}\right| \Psi_{n}^{T}(\beta)\right\rangle=1$. This reference energy is different from the one commonly used to obtain the ground state. In practice, $E_{n}^{X}$ depends on the Slater determinant expansion $\chi_{n}^{X}(\mathbf{R})$ used to construct the guiding wave function and contains the relevant information required to calculate thermodynamical averages.

Using Eqs (2) and (7), one can easily obtain

$$
\begin{aligned}
\frac{\partial}{\partial \beta} f_{n}(\mathbf{R}, \beta) & =\Psi_{n}^{T *}(\mathbf{R}) \frac{\partial}{\partial \beta}\left[\Psi_{n}^{T}(\mathbf{R}, \beta) e^{\beta E_{n}^{X}}\right] \\
& =-\Psi_{n}^{T *}(\mathbf{R})\left[\left(\hat{\mathcal{H}}-E_{n}^{X}\right) \Psi_{n}^{T}(\mathbf{R}, \beta) e^{\beta E_{n}^{X}}\right] \\
& =-\left[E_{n}^{T}(\mathbf{R}, \beta)-E_{n}^{X}\right] f_{n}(\mathbf{R}, \beta)
\end{aligned}
$$

with

$$
\begin{aligned}
E_{n}^{T}(\mathbf{R}, \beta)= & \frac{\hat{\mathcal{H}} \Psi_{n}^{T}(\mathbf{R}, \beta)}{\Psi_{n}^{T}(\mathbf{R}, \beta)} \\
= & -\frac{1}{2} \sum_{j}^{N_{e}} \frac{\nabla_{j}^{2} \Phi_{n}^{T}(\mathbf{R}, \beta)}{\Phi_{n}^{T}(\mathbf{R}, \beta)} \\
+ & \frac{1}{2} \sum_{j}^{N_{e}}\left|\mathbf{A}_{j}+\nabla_{j} \phi_{n}(\mathbf{R})\right|^{2}+V(\mathbf{R}, \beta) \\
- & \mathbf{i} \sum_{j}^{N_{e}}\left\{\frac{\nabla_{j} \Phi_{n}^{T}(\mathbf{R}, \beta)}{\Phi_{n}^{T}(\mathbf{R}, \beta)} \cdot\left[\mathbf{A}_{j}+\nabla_{j} \phi_{n}(\mathbf{R})\right]\right. \\
& \left.\quad+\frac{\nabla_{j} \cdot\left[\mathbf{A}_{j}+\nabla_{\mathbf{j}} \phi_{n}(\mathbf{R})\right]}{2}\right\} .
\end{aligned}
$$

In order to perform an importance sampling using $\Phi_{n}^{T}(\mathbf{R})$ as a guiding wave function (as in Refs. 10 and 31), it is convenient to express the kinetic energy in terms of $\Phi_{n}^{T}(\mathbf{R})$. The term including $\nabla_{j}^{2} \Phi_{n}^{T}(\mathbf{R}, \beta) / \Phi_{n}^{T}(\mathbf{R}, \beta)$ can be rewritten using the identity $\underline{\underline{10.29 .55}}$

$$
\begin{aligned}
\frac{\nabla_{j}^{2} \Phi_{n}^{T}(\mathbf{R}, \beta)}{\Phi_{n}^{T}(\mathbf{R}, \beta)}= & \frac{\nabla_{j}^{2} f_{n}(\mathbf{R}, \beta)}{f_{n}(\mathbf{R}, \beta)}+\frac{\nabla_{j}^{2} \Phi_{n}^{T}(\mathbf{R})}{\Phi_{n}^{T}(\mathbf{R})} \\
& -\frac{\nabla_{j} \cdot\left[f_{n}(\mathbf{R}, \beta) \mathbf{F}_{Q}^{j}\right]}{f_{n}(\mathbf{R}, \beta)},
\end{aligned}
$$


where

$$
\mathbf{F}_{Q}^{j}=\nabla_{j} \ln \left|\Phi_{n}^{T}(\mathbf{R})\right|^{2}
$$

Note that Eqs. (13) and (14) are valid as long as $\Phi_{n}^{T}(\mathbf{R}) \neq 0$ and $f_{n}(\mathbf{R}, \beta) \neq 0$. In practice, any divergence of $\mathbf{F}_{Q}^{j}$ at the nodes enforces $f_{n}(\mathbf{R}, \beta)$ to be zero. Figure 1 shows that complex antisymmetric wave functions can be constructed so that they have nodes only at points $\mathbf{R}$ with $\mathbf{r}_{i}=\mathbf{r}_{j}$. In this case the nodal error is avoided but errors in the phase introduce a phase shift ${ }^{31.42}$ and a complex contribution to $E_{n}^{L}(\mathbf{R}, \beta)$. However, for complex wave functions without zeros, Eq. (13) is always valid, except at the coincidental points (if cusp conditions are not satisfied). To satisfy Eq. (13) at the coincidental points, a Jastrow factor is introduced in Eq. (4). While using complex wave functions involves some complications, the advantage is that the evolution in imaginary time $\beta$ describes the thermodynamical properties with $\beta^{-1}=k_{B} T$. However, going beyond the fixed-phase approximation ${ }^{31.56}$ is required to obtain the thermodynamics. In this work the phase is not "released" in the same sense of Ref. ${ }^{33}$, it is only free within the small subspace.

Replacing Eq. (13) into Eq (12) and then into Eq. (11) one obtains

$$
\begin{aligned}
\frac{\partial f(\mathbf{R}, \beta)}{\partial \beta}= & \frac{1}{2} \sum_{j}^{N_{e}}\left\{\nabla_{j}^{2} f_{n}(\mathbf{R}, \beta)-\nabla_{j} \cdot\left[f_{n}(\mathbf{R}, \beta) \mathbf{F}_{Q}^{j}\right]\right\} \\
& -\left[E_{n}^{L}(\mathbf{R}, \beta)-E_{n}^{X}\right] f_{n}(\mathbf{R}, \beta),
\end{aligned}
$$

with $E_{n}^{L}(\mathbf{R}, \beta)$ where

$$
\begin{aligned}
E_{n}^{L}(\mathbf{R}, \beta)= & -\frac{1}{2} \sum_{j}^{N_{e}} \frac{\nabla_{j}^{2} \Phi_{n}^{T}(\mathbf{R})}{\Phi_{n}^{T}(\mathbf{R})} \\
& +\frac{1}{2} \sum_{j}^{N_{e}}\left|\mathbf{A}_{j}+\nabla_{j} \phi_{n}(\mathbf{R})\right|^{2}+V(\mathbf{R}, \beta) \\
& -\mathbf{i} \sum_{j}^{N_{e}}\left\{\frac{\nabla_{j} \Phi_{n}^{T}(\mathbf{R}, \beta)}{\Phi_{n}^{T}(\mathbf{R}, \beta)} \cdot\left[\mathbf{A}_{j}+\nabla_{j} \phi_{n}(\mathbf{R})\right]\right. \\
& \left.+\frac{\nabla_{j} \cdot\left[\mathbf{A}_{j}+\nabla_{\mathbf{j}} \phi_{n}(\mathbf{R})\right]}{2}\right\} .
\end{aligned}
$$

That is, the local energy now depends on two guiding functions (i) $\Phi_{n}^{T}(\mathbf{R})$, and (ii) $\Phi_{n}^{T}(\mathbf{R}, \beta)$ which is an approximation that must be obtained and improved for $\beta \neq 0$.

The use of complex valued guiding functions originates the gradients $\nabla_{j} \phi_{n}(\mathbf{R})$ that appear in the local energy in Eq. (16). Their contribution prevents the result from reaching the bosonic 
solution and enforces an upper bound on the fermionic ground state 31 In addition, the contribution of $\nabla_{j} \Phi_{n}^{T}(\mathbf{R}, \beta)$ must be taken into account in the presence of a magnetic field (see term between the \{\} , when $\mathbf{A}_{j} \neq 0$ ), even when using a real-bosonic guiding wave function with $\nabla_{j} \phi_{n}(\mathbf{R})=0$.

A locality approximation ${ }^{57}$ has been used in the past when a non-local pseudo potential is included in $\hat{\mathcal{H}}$ in the potential term $\hat{V}(\mathbf{R})$. It consists in replacing $V(\mathbf{R}, \beta)=\frac{\hat{V}(\mathbf{R}) \Psi_{n}^{T}(\mathbf{R}, \beta)}{\Psi_{n}^{T}(\mathbf{R}, \beta)}$ by $V(\mathbf{R}, 0)$ in Eq. (12). Since $V(\mathbf{R}, \beta) \rightarrow V(\mathbf{R}, 0)$ for $\beta \rightarrow 0$, we will use the locality approximation in the first iteration. However, we will improve it in subsequent iterations (see Appendix A).

A local-time approximation, analogous to the locality approximation, 57 , was introduced in Ref. 42 to estimate the ratio

$$
\frac{\nabla_{j} \Phi_{n}^{T}(\mathbf{R}, \beta)}{\Phi_{n}^{T}(\mathbf{R}, \beta)}=\frac{\nabla_{j} \Phi_{n}^{T}(\mathbf{R})}{\Phi_{n}^{T}(\mathbf{R})}+\nabla_{j} \ln \left[\frac{\Phi_{n}^{T}(\mathbf{R}, \beta)}{\Phi_{n}^{T}(\mathbf{R})}\right]
$$

One can neglect the last term in Eq. (17) for $\beta \rightarrow 0$ where $\Phi_{n}^{T}(\mathbf{R}, \beta) / \Phi_{n}^{T}(\mathbf{R}) \rightarrow 1$. In the present work we will use the local-time approximation only in the first iteration. For subsequent iterations we improve the evaluation of Eq. (17) by using the sampling of the dependence in $\beta$ of $\Phi_{n}^{T}(\mathbf{R}, \beta)$ obtained in the previous iteration.

The locality and local-time approximations have little impact in optimization methods that focus on eigenstates because the dependence on $\beta$ of $\Psi_{n}^{T}(\mathbf{R}, \beta)$ is minimized when the optimization progresses as $\Psi_{n}^{T}(\mathbf{R}) \rightarrow \Psi_{n}(\mathbf{R})$. Going beyond these approximations is required, however, to circumvent the nodes of the eigenstates with complex wave functions. Fortunately, optimization in the small subspace allows an easy sampling of the $\beta$ dependence. The approach is exact if the big basis is large enough and if enough statistical data is collected ${ }^{58}$ as $\ell \rightarrow \infty$.

Circumventing the nodes with complex wave functions is necessary in this case because, in standard DMC calculations using real-valued wave functions with nodes, if any walker crosses a node, it is either killed ${ }^{10}$ or the move is rejected. ${ }^{59}$ This introduces an artificial divergent potential at the nodal surface, which adds a kink at the node (a step for the rejection case). Since there is a one-to-one correspondence between energy of one eigenstate and its nodes, $\underline{60}$ eigenstates with different energies must have different nodes. As a consequence, two real wave wave functions that approach different eigenstates introduce different nodal potentials. Since the fixed-node Hamiltonian is different for different eigenstates, and affect the dynamics at the node in the evolution in imaginary time, the $\beta$ dependence obtained using the fixed-node approximation will not describe the thermodynamics even if the exact nodes of the ground state are provided. 


\section{Differences with other DMC-like projection methods}

The implementation of this method follows essentially the same approach developed for DMC or SHDMC, with some key numerical changes.

Equation (15) is identical to Eq. (1) in Ref. 10 except for the local energy, which now has an explicit dependence in $\beta$. Unlike Eq. (13) in Ref. 42, Eq. (15) is now valid for $\beta \geq 0$. As in Ref 10, Eq (15) describes the evolution of an ensemble. Each member of the ensemble of configurations $\mathbf{R}_{i}$ (walker) undergoes (i) a random diffusion and (ii) drifting by the quantum force $\sum_{j} \mathbf{F}_{Q}^{j}$ (which depends only on $\Phi_{T}(\mathbf{R})$ and not on the phase). Following Ref. 42, (iii) each walker carries a complex phase factor. In a nonbranching algorithm, the complex weight of the walkers is multiplied by $\exp \left\{-\left[E_{n}^{L}(\mathbf{R}, \beta)-E_{n}^{X}\right] \delta \beta\right\}$ at every diffusion step. In contrast with Ref. 33, the phase factor of the walkers starts in 1 and evolves towards the difference between the guiding phase and the phase of $\Psi(\mathbf{R}, \beta)$, while in the release phase approach the initial phase of each walker depends on the initial positions of the walkers $\mathbf{R}_{i}$ but remains constant in $\beta$.

If the $\chi_{n}^{X}(\mathbf{R})$ are linear combinations of antisymmetric functions, with arbitrary complex coefficients, the $\Phi_{n}^{T}(\mathbf{R})$ in Eq. (16) do not have nodal surfaces (see Fig. 1). Therefore, $\mathbf{F}_{q}$ is not divergent, but at the coincidental points.

All walkers must add the same inverse temperature $\beta$ after $k$ steps. The standard time-step correction to minimize time step errors at the nodes [Eq. (33) in Ref. 59] is not strictly necessary since the divergences in $\mathbf{F}_{q}$ are removed. If one introduces it, one must readjust $\delta \beta$ during the time evolution so that all the walkers add up to the same $\beta$. For the same reason, the standard accept/reject scheme that enforces detailed balance ${ }^{55}$ is modified: other moves are retried after rejection until a move is accepted.

\section{E. Calculation of the partition function}

This section shows that the partition function can be obtained as $Z(\beta) \simeq \sum_{n=0}^{M_{S}-1} e^{-\beta E_{n}^{S}}$ where the $E_{n}^{S}$ are reference energies instead of eigen energies.

Numerically, it is convenient to start the calculation with a distribution of walkers proportional to $f_{n}(\mathbf{R}, 0)=\left|\Phi_{n}^{T}(\mathbf{R})\right|^{2}$. In the importance sampling approach $\underline{10}$ setting the second line of Eq. (15) equal to zero, provides an equilibrium distribution proportional to $\left|\Phi_{n}^{T}(\mathbf{R})\right|^{2}$.

As in the DMC and SHDMC methods, the evolution in inverse temperature $\beta$ is discretized 
into $k$ finite steps $\delta \beta=\beta / k$. Following the SHDMC approach ${ }^{40-42,47}$ the weighted distribution of the walkers can be written as

$$
f_{n}(\mathbf{R}, k \delta \beta)=\lim _{N_{c} \rightarrow \infty} \frac{1}{N_{c}} \sum_{i=1}^{N_{c}} W_{i} \delta\left(\mathbf{R}-\mathbf{R}_{i}\right) .
$$

In Eq. (18), $\mathbf{R}_{i}$ corresponds to the position of the walker $i$, and $N_{c}$ is the number of equilibrated configurations. The complex weights $W_{i}$ are given by

$$
W_{i}=e^{-\left[E_{i}^{k}-E_{n}^{X}\right] k \delta \beta}
$$

with

$$
E_{i}^{k}=\frac{1}{k} \sum_{j=0}^{k-1} E_{n}^{L}\left(\mathbf{R}_{i}^{-j}\right)
$$

Where $k$ is a number of steps and $E_{L}\left(\mathbf{R}_{i}^{-j}\right)$ is the previous value of the local energy obtained $j$ steps $\delta \beta$ earlier for the walker $i$.

The evolution in inverse temperature $\beta$ of the guiding wave function $\Psi_{n}^{T}(\mathbf{R}, \beta)$ can be written, without loss of generality, as

$$
\Psi_{n}^{T}(\mathbf{R}, \beta)=e^{-\beta E_{n}^{X}} e^{-J(\mathbf{R})}\left[\chi_{n}^{X}(\mathbf{R})+\delta \chi_{n}^{X}(\mathbf{R}, \beta)\right]
$$

That is, the product of an average decay factor $e^{-\beta E_{n}^{X}}$ times the Slater determinant part. The Slater part is given by the the one at $\beta=0$ plus an orthogonal displacement $\delta \chi_{n}^{X}(\mathbf{R}, \beta)$. The $X$ in term $\delta \chi_{n}^{X}(\mathbf{R}, \beta)$ denotes the explicit dependence on $\chi_{n}^{X}(\mathbf{R})$. At least one overlap $\left\langle\chi_{m}^{X} \mid \delta \chi_{n}^{X}, \beta\right\rangle$ must be different from zero for $n \neq m$, if $\Psi_{n}^{T}(\mathbf{R})$ is not an eigenstate.

Using Eq. (21) we can correct equation (16) beyond the locality and local-time approximations. The displacement $\delta \chi_{n}^{X}(\mathbf{R}, \beta)$ can be sampled from the DMC run as follows: From Eqs. (7), (10) and (18), one can obtain

$$
\begin{aligned}
e^{-J(\mathbf{R})} \delta \chi_{n}^{X}(\mathbf{R}, \beta) & =\left\langle\mathbf { R } \left|\left[e^{\beta E_{n}^{X}}\left|\Psi_{n}^{T}(\beta)\right\rangle-\left|\Psi_{n}^{T}\right\rangle\right]\right.\right. \\
& =\left\langle\mathbf{R}\left|\left[e^{-\beta\left(\hat{\mathcal{H}}-E_{n}^{X}\right)}-1\right] e^{-\hat{J}}\right| \chi_{n}^{X}\right\rangle \\
& =\frac{\left[f_{n}(\mathbf{R}, \beta)-f_{n}(\mathbf{R}, 0)\right]}{\Psi_{n}^{T *}(\mathbf{R})} \\
& \simeq \frac{1}{N_{c}} \sum_{i=1}^{N_{c}} e^{J(\mathbf{R})} \frac{\left[W_{i}-1\right]}{\chi_{n}^{X *}(\mathbf{R})} \delta\left(\mathbf{R}-\mathbf{R}_{i}\right) .
\end{aligned}
$$


Within the subspace spanned by the basis $\left\{\chi_{n}^{S}(\mathbf{R})\right\}$, the identity operator $\hat{E}$ is given by

$$
\left\langle\mathbf{R}^{\prime}|\hat{E}| \mathbf{R}\right\rangle=\sum_{m=0}^{M_{S}-1} e^{-J\left(\mathbf{R}^{\prime}\right)} \chi_{m}^{S}\left(\mathbf{R}^{\prime}\right) \chi_{m}^{S *}(\mathbf{R}) e^{J(\mathbf{R})} .
$$

Applying Eq. (25) to both sides of Eq. (24), and integrating over R, one can easily obtain an expression of the diffusion displacement within the basis $\left\{\left|\chi_{n}^{S}\right\rangle\right\}$ as

$$
\delta \chi_{n}^{X}(\mathbf{R}, \beta)=\sum_{m=0}^{M_{S}-1} \lambda_{n}^{m}(\beta) \chi_{m}^{S}(\mathbf{R})
$$

with

$$
\lambda_{n}^{m}(\beta)=\frac{1}{N_{c}} \sum_{i=1}^{N_{c}} e^{2 J\left(\mathbf{R}_{i}\right)} \frac{\chi_{m}^{S *}\left(\mathbf{R}_{i}\right)}{\chi_{n}^{X *\left(\mathbf{R}_{i}\right)}}\left[W_{i}-1\right]
$$

where $N_{c}=\sum_{i=1}^{N_{c}} W_{i}$.

Using $X=S$ in Eqs. (18) -(21) one can prove that

$$
\begin{aligned}
\left\langle\chi_{n}^{S}|\hat{\mathcal{U}}| \chi_{m}^{S}\right\rangle & =\int \mathbf{d} \mathbf{R} e^{J(\mathbf{R})} \chi_{n}^{S *}(\mathbf{R}) \Psi_{m}^{T}(\mathbf{R}, \beta) \\
& =e^{-\beta E_{n}^{S}}\left[\delta_{n, m}+\int \mathbf{d} \mathbf{R} \chi_{n}^{S *}(\mathbf{R}) \delta \chi_{m}^{S}(\mathbf{R}, \beta)\right] \\
& =e^{-\beta E_{n}^{S}}\left(\delta_{n, m}+\lambda_{n}^{m}\right)
\end{aligned}
$$

with $\hat{\mathcal{U}}$ having the structure of the transcorrelated method ${ }^{61}$

$$
\hat{\mathcal{U}}=e^{\hat{J}} e^{-\beta \hat{\mathcal{H}}} e^{-\hat{J}}
$$

We use condition $\lambda_{n}^{n}=0$ [See Eq (27)] to determine the value of $E_{n}^{S}$. In practice, we adjust the reference energy of the guiding functions every iteration as $e^{-\beta E_{n}^{X, \ell+1}}=e^{-\beta E_{n}^{X, \ell}}\left(\delta_{n, m}+\lambda_{n}^{m}\right)$.

Since $\operatorname{tr}\left(e^{-\beta \hat{\mathcal{H}}}\right)=\operatorname{tr}(\hat{\mathcal{U}})$, the contribution to the Helmholtz free energy of the small subspace is given by

$$
F(\beta)=-\frac{1}{\beta} \ln \left[\sum_{n=0}^{M_{S}-1} e^{-\beta E_{n}^{S}}\right],
$$

where the expression inside the brackets is the partition function $Z(\beta)$.

In general for an arbitrary guiding function $\Psi_{m}^{T}(\mathbf{R})$, the variance will grow with $M_{S}$. An energy span larger than $\beta^{-1}$ must be retained in the basis to calculate thermodynamical properties. 
Arbitrary trial wave functions spanned by this space might have significant variance in the walkers weights. To reduce the variance we use guiding functions that are approximately linear combinations of a pair of neighboring eigenstates.

When using guiding functions that are different from the small basis functions, the contributions to the trace of the density matrix in the small basis can be obtained with

$$
e^{-\beta E_{n}^{S}}=\sum_{i}\left[\left|\left\langle\chi_{i}^{X} \mid \chi_{n}^{S}\right\rangle\right|^{2}+\left\langle\chi_{i}^{X} \mid \chi_{n}^{S}\right\rangle\left\langle\chi_{n}^{S} \mid \delta \chi_{i}^{X}\right\rangle\right] e^{-\beta E_{i}^{X}} .
$$

The details of the derivation are in Appendix B,

In a recent paper, Mazzola, Zen and Sorella $a^{\frac{5}{5}}$ proved that

$$
\left\langle\Psi_{n}^{T}\left|e^{-\beta \hat{\mathcal{H}}}\right| \Psi_{n}^{T}\right\rangle \geq e^{-\beta\left\langle\Psi_{n}^{T}|\hat{\mathcal{H}}| \Psi_{n}^{T},\right\rangle} .
$$

Ref. 5 used the righthand side of Eq. (32) to approximate the free energy obtaining a lower bound for $F(\beta)$. Reference 5 can be considered a VMC approach to the evaluation of the free energy. That approximation becomes exact if all the $\left|\Psi_{n}^{T}\right\rangle$ are eigenstates of $\hat{\mathcal{H}}$. However, that method is very poor for an arbitrary random guiding function. In the present approach, we go beyond Ref. 5 by evaluating the lefthand side of Eq. (32) directly using DMC.

In many situations, the excitations of a mean field method based on approximations DFT might be good enough to obtain the low energy thermodynamical properties using Eq. (30). If that were the case, at least two DMC runs for each function of the basis are required. One to obtain the $\beta$ dependence and a second to evaluate the reference energies beyond the local-time approximation. However, in the so-called highly correlated materials, usual approximations of DFT fail to describe the low energy physics. In those cases a method that could optimize the basis is more important. That method is described in the following subsections.

\section{F. The first iteration: Construction of the first small basis $\left\{\left|\chi_{n}^{S, 1}\right\rangle\right\}$}

While the present approach will optimize the basis from any starting basis set, the calculation will be more efficient starting from a good basis. A procedure to generate a good starting set is described here.

The only restriction for the small basis $\left\{\left|\chi_{n}^{S}\right\rangle\right\}$ is to avoid the nodes associated with real wave functions. In this work we choose the initial basis with a Lanczos-like procedure combined with the SHDMC approach. 
The big subspace basis set $|m\rangle$ is constructed by symmetry constrained functions (linear combinations of Slater determinants with the same symmetry of the ground state) ordered with increasing mean field energy.

We choose the first basis function of the small subspace to be

$$
\left|\Psi_{0}^{S}\right\rangle=\frac{1}{\sqrt{2}}(|0\rangle+\mathbf{i}|1\rangle)
$$

being $|0\rangle$ and $|1\rangle$ the ground and first excited states of a non-interacting solution of the system.

Using Eq. (33) as guiding function in Eqs. (22)-(24) and replacing $\chi_{m}^{S}(\mathbf{R})$ by $\langle\mathbf{R} \mid m\rangle$ in Eq. (25), but not on $\Psi_{n}^{T}(\mathbf{R})$, we obtain an expression similar to Eq. (26)

$$
\delta \tilde{\chi}_{n}^{S}(\mathbf{R})=\left\langle\mathbf{R} \mid \delta \tilde{\chi}_{n}^{S}\right\rangle=\tilde{\sum_{m=0}} c_{n}^{m}\langle\mathbf{R} \mid m\rangle .
$$

The tilde in $\delta \tilde{\chi}_{n}^{S}(\mathbf{R})$ means that the expansion is in the big basis $\{|m\rangle\}$ with

$$
c_{n}^{m}(\beta)=\frac{1}{N_{c}} \sum_{i=1}^{N_{c}} e^{2 J\left(\mathbf{R}_{i}\right)} \frac{\left\langle m \mid \mathbf{R}_{i}\right\rangle}{\chi_{n}^{S *}\left(\mathbf{R}_{i}\right)}\left[W_{i}-1\right] .
$$

The symbol $\tilde{\sum}$ in Eq. (34) means that the sum is restricted to the coefficients $c_{n}^{m}$ with an error bar smaller than $25 \%$ of the absolute value (this is the standard recipe of the SHDMC algorithm ${ }^{40}$ ).

We define the next basis function $\left|\chi_{n+1}^{S}\right\rangle$ recursively as

$$
\begin{aligned}
\left|\chi_{n+1}^{S}\right\rangle & =\frac{1}{\mathcal{N}_{n+1}} \hat{P}_{n+1}\left|\delta \tilde{\chi}_{n}^{S}\right\rangle \text { with } \\
\hat{P}_{n} & =1-\sum_{m=0}^{n-1}\left|\chi_{m}^{S}\right\rangle\left\langle\chi_{m}^{S}\right|
\end{aligned}
$$

where $\mathcal{N}_{n+1}$ is a normalization constant. Equations (37) and (36) mean that $\left|\chi_{n}^{S}\right\rangle$ is the projection of the displacement $\left|\delta \tilde{\chi}_{n+1}^{S}\right\rangle$ orthogonal to the subspace spanned by the $n$ basis functions found previously.

One repeats this procedure until a basis of $M_{S}$ functions $\left\{\left|\chi_{n}^{S}\right\rangle\right\}$ is constructed. This Lanczoslike procedure grants that the initial small subspace basis times the Jastrow factor has a large projection onto the lowest-energy eigenstates of $\hat{\mathcal{H}}$ or the largest eigenstates of $e^{-\beta \hat{\mathcal{H}}}$.

Since the evolution in inverse temperature $\beta$ is not known during the initialization step, we use the local-time approximation discussed in the previous section. However, once a basis is generated, we can go beyond the local-time approximation in successive iterations. Note that by construction 
any $\chi_{n}^{S}(\mathbf{R}, \beta)$ can be approximated as a linear combination of the basis function $\chi_{m}^{S}(\mathbf{R})$ with $m<n+1$, since the finite temperature projection of one wave function of the basis into the other was used to construct the small basis. The details on how to approximate the evolution in $\beta$ are in Appendix $\mathrm{A}$

\section{G. Systematic improvement of the small basis $\left\{\left|\chi_{n}^{S}\right\rangle\right\}$}

One of the goals of this work is to obtain a much smaller basis of $M_{S}$ functions $\left\{\left|\chi_{n}^{S}\right\rangle\right\}$ than $\{|m\rangle\}$, the big set of $N_{B}$ basis functions. The small basis $\left\{\left|\chi_{n}^{S}\right\rangle\right\}$ should retain the lowest energy physics of $\hat{\mathcal{H}}$ and $e^{-\beta \hat{\mathcal{H}}}$. While for some purposes (e.g. the ground state calculation), the initial basis set described in the previous section might be enough, in this subsection we describe how to further optimize the small basis so it better describes the low-energy excitations of $\hat{\mathcal{H}}$ and thermodynamical properties.

Note that $\chi_{n}^{S}(\mathbf{R}, \beta)=e^{\hat{J}} e^{-\beta \hat{\mathcal{H}}} e^{-\hat{J}} \chi_{n}^{S}(\mathbf{R})$ will converge to the antisymmetric part of the ground state wave-function as $\beta \rightarrow \infty$. In order to avoid every state in the basis collapsing to the same function we (i) remove the projection into the other states of the basis, (ii) add the diffusion displacement orthogonal to the small subspace, and (iii) perform a GramSchmidt orthogonalization as follows:

$$
\begin{aligned}
& \left|\chi_{0}^{S, \ell+1}\right\rangle=\left|\chi_{0}^{S, \ell}\right\rangle+\left|\delta \tilde{\chi}_{0}^{S}\right\rangle-\left|\delta \chi_{0}^{S}(\beta)\right\rangle \\
& \left|\chi_{n}^{S, \ell+1}\right\rangle=\hat{P}_{n}^{\ell+1}\left[\left|\chi_{n}^{S, \ell}\right\rangle+\left|\delta \tilde{\chi}_{n}^{S}\right\rangle-\left|\delta \chi_{1}^{S}(\beta)\right\rangle\right] n>0
\end{aligned}
$$

with $\hat{P}_{n}^{\ell+1}$ given by Eq. (37) replacing $\chi_{m}^{S}$ by $\chi_{m}^{S, \ell+1}$. Note in Eq. (38) that $\left|\delta \tilde{\chi}_{n}^{S}\right\rangle$ given by Eq. (34) is a direct projection of the diffusion displacement into the big basis of SCFs $\{|m\rangle\}$, whereas $\left|\delta \chi_{n}^{S}(\beta)\right\rangle$ given by Eq. (26) is an indirect projection (since the $\left|\delta \chi_{n}^{S}(\beta)\right\rangle$ are projected into the small basis $\left\{\left|\chi_{n}^{S}\right\rangle\right\}$ which in turn are linear expansions of functions that belong to $\left.\{|m\rangle\}\right)$. The difference $\left|\Delta \tilde{\chi}_{n}^{S}\right\rangle=\left|\delta \tilde{\chi}_{n}^{S}\right\rangle-\left|\delta \chi_{n}^{S}(\beta)\right\rangle$ is by construction orthogonal to the small subspace. Accordingly, it describes the decay of the small subspace basis into the eigenstates with the lowests energies.

\section{ALGORITHM}

The goal of this algorithm is to optimize a minimal basis $\left\{\left|\chi_{n}^{S}\right\rangle\right\}$ to span the lowest energy excitations of $\hat{\mathcal{H}}$ (equivalently the eigenstates of $e^{-\beta \hat{\mathcal{H}}}$ with the largest eigenvalues). That basis 
can be used to calculate finite temperature expectation values of thermodynamical properties and accelerate the calculation of the ground and lower excited states. In this section we summarize how the theory described in detail earlier can be implemented.

Initialization: the small basis $\left\{\left|\chi_{n}^{S}\right\rangle\right\}$, a set of orthogonal linear combinations of many-body functions $|m\rangle$ is constructed using the procedure described in $\amalg F$ Once this procedure is concluded, a set of linearly independent guiding functions $e^{-\hat{J}}\left|\chi_{n}^{V}\right\rangle$ is constructed as linear combinations of pairs of approximated eigenstates of $\hat{\mathcal{U}}$.

Subsequently, we can use for the evaluation of the local energy $E_{n}^{L}(\mathbf{R}, \beta)$ an approximate dependence of the guiding functions in $\beta$ given by Eq. (C2).

Basis update iteration: each iteration $\ell$ is composed by (1) a parallel diffusion of intermediate functions $\left|\chi_{n}^{V}\right\rangle$. (2) A linear transformation to obtain $\left|\delta \tilde{\chi}_{n}^{S}\right\rangle$ and $\left|\delta \chi_{n}^{S}\right\rangle$. (3) Recalculation of $\hat{\mathcal{U}}$ in the small basis. (4) Update of the small basis for the next iteration $\left|\tilde{\chi}_{n}^{S, \ell+1}\right\rangle$. (5) Update of the intermediate functions $\left|\chi_{n}^{V, \ell+1}\right\rangle$. (6) Finally, the algorithm decides to increase the number of samples in the next iteration or not. These six steps are repeated recursively.

The individual steps of the iteration are described below in more detail:

(1) Parallel diffusion: Each displacement

$$
\left|\delta \chi_{n}^{V}\right\rangle=e^{\hat{J}}\left[e^{-\beta \hat{\mathcal{H}}}-1\right] e^{-\hat{J}}\left|\chi_{n}^{V}\right\rangle
$$

is projected into the small basis $\left\{\left|\chi_{n}^{S}\right\rangle\right\}$ and the big basis $\{|m\rangle\}$ using Eqs. (27) and Eq. (35) respectively replacing $\chi_{n}^{X}(\mathbf{R})$ by $\chi_{n}^{V}(\mathbf{R})$.

Each diffusion contains $M_{b}$ sampling subblocks. For each sampling subblock, uncorrelated walker positions are generated from the previous one with a VMC algorithm. Next, DMC is run for $k$ steps with a shorter time step $\delta \beta$. The coefficients of $\left|\delta \tilde{\chi}_{n}^{V}\right\rangle$ and $\left|\delta \chi_{n}^{V}\right\rangle$ are sampled at the end of each subblock using Eqs. (27) and (35). Statistical data is collected for $M_{b}$ subblocks for each parallel diffusion before an update of the small basis.

(2) Linear transformation: The $\left|\delta \tilde{\chi}_{n}^{S}\right\rangle$ and $\left|\delta \chi_{n}^{S}\right\rangle$ can be constructed in terms of $\left|\delta \tilde{\chi}_{n}^{V}\right\rangle$ and $\left|\delta \chi_{n}^{V}\right\rangle$ using Eq. (B1) replacing the super index $X$ by $V$.

(3) Calculation of $\hat{\mathcal{U}},\left\{\left|\Psi_{n}^{U, \ell+1}\right\rangle\right\}$, and $\left\{\left|\Psi_{n}^{V, \ell+1}\right\rangle\right\}$ : A matrix representation of $\hat{\mathcal{U}}$ is obtained in the small subspace $\left\{\left|\Psi_{n}^{U, \ell}\right\rangle\right\}$, using Eqs. (27), (28) and (B1). The left and right eigenvectors are obtained by diagonalizing $\hat{\mathcal{U}}$.

(4) Update of the small Basis: Equation (38) is used to improve the small basis.

(5) We perform the correspondence $\chi_{n}^{S, \ell} \rightarrow \chi_{n}^{S, \ell+1}$ (see Appendix $\mathrm{A}$ ) and construct the basis 
$\left\{\left|\chi_{n}^{U, \ell+1}\right\rangle\right\}$, and $\left\{\left|\chi_{n}^{V, \ell+1}\right\rangle\right\}$ with the coefficients of the eigenvectors of $\hat{\mathcal{U}}$ in the iteration $\ell$.

(6) Updating $M_{b}$ : At first, the number of sampling subblocks $M_{b}$ is set to a small number (e.g. $\left.M_{b}=3\right)$. When the noise is dominant $\sum_{n}\left\langle\Delta \tilde{\chi}_{n}^{S, \ell+1} \mid \Delta \tilde{\chi}_{n}^{S, \ell}\right\rangle \leq 0$, we increase $M_{b}$ by a factor larger than 1. As a result, the total number of configurations $N_{c}$ sampled increases as the iteration $\ell$ increases and the statistical error is reduced. Hence, as the statistics are improved the number of basis functions retained in the expansion Eq. (25) increases over time.

(7) We use Eq. (30) to calculate thermodynamical properties.

\section{RESULTS IN MODEL CALCULATIONS}

This section describes the results obtained for a model system with an applied magnetic field. The results are compared with configuration interaction (CI) calculations in the same model used in Refs. 40,47,60 and 42. The lowest-energy eigenstates were found for two polarized electrons $(J=1)$ moving in a two-dimensional square with a side length 1 and a repulsive interaction potential of the form $\hat{V}\left(\mathbf{r}, \mathbf{r}^{\prime}\right)=8 \pi^{2} \gamma \cos \left[\alpha \pi\left(x-x^{\prime}\right)\right] \cos \left[\alpha \pi\left(y-y^{\prime}\right)\right]$ with $\alpha=1 / \pi$ and $\gamma=4$.

The main advantage of the model is that fully converged CI calculations can be performed which are nearly analytical. In order to perform the CI calculations the many-body wave function of the small basis $\left.\left\{\chi_{n}^{S}\right\rangle\right\}$ are spanned in a basis of functions $\{|m\rangle\}$ that are eigenstates of the noninteracting system. They are linear combinations of functions of the form $\prod_{\nu} \sin \left(m_{\nu} \pi x_{\nu}\right)$ with $m_{\nu} \leq 7$. Converged CI calculations were performed to obtain a nearly exact expression of the lowest energy states of the system $\left|\Psi_{n}\right\rangle=\sum_{m} a_{m}^{n}|m\rangle$. The matrix elements involving the magnetic vector potential A (in the symmetric gauge) were calculated analytically. The result of the CI calculations were used to evaluate the partition functions and to quantify the convergence of the basis.

The same basis used to construct the CI Hamiltonian is used as the big basis to test our finite temperature version of SHDMC. All the calculations reported are with $J(\mathbf{R})=0$, which increases the statistically noise, makes the test more difficult and facilitates the comparison with the CI results. The results presented here are a proof of principle on the validity of the algorithm, which is necessary before requesting and using the massive amount of computing time required for realistic finite temperature calculations in solids. While clearly a demonstration in a realistic system is required in the future, a comparison with an exact model is the first essential step to validate the scheme. This includes not only the value obtained for the partition function but also a detailed 
analysis of the convergence of the basis.

In the absence of magnetic fields there are two degenerate solutions: one that transforms line $x$, and the other that transform like $y$. This degeneracy is broken with a magnetic field. The eigenstates transform like $x+\mathbf{i} y$ and $x-\mathbf{i} y$. Figure 2 shows the evolution of $Z_{+}(\beta)=\operatorname{tr}+(\hat{\mathcal{U}})$ of the model system with $M_{S}=20$. The subindex " + " in $Z_{+}$and $t r_{+}$means that the results of Fig. (2) were obtained considering only the subspace of the Hamiltonian that transforms like $x+\mathbf{i} y$. The calculation of thermodynamical properties requires, however, the inclusion of all possible symmetries of the wave function, which implies that $Z_{-}$, the trace in a small basis that transforms as $x-\mathbf{i} y$, should also be added. In order to calculate $Z_{+}(\beta)$ we have defined the zero of energy to be the ground state of the CI. The calculations were run using $\delta \beta=0.00002$ and $\beta=0.004$. We have used a magnetic field of $B=0.6283$.

Figure 2 shows the value of $Z_{+}(\beta)$, calculated with different methods, relative to the full CI value obtained with the $M_{S}=20$ lowest eigenvalues. The blue cycles were obtained with SHDMC using Eq. (30). The red rhombi were obtained by evaluating $Z_{+}(\beta)=\sum_{n, m}\left|\left\langle\Psi_{m}^{T} \mid \Psi_{n}\right\rangle\right|^{2} e^{-\beta E_{n}}$, being the $\left|\Psi_{n}\right\rangle$ and $E_{n}$ the eigenvectors and eigenvalues of the full CI. The empty squares were obtained as $Z_{+}(\beta) \simeq \sum_{n} e^{-\beta\left\langle\left|\Psi_{n}^{T}\right| \hat{\mathcal{H}} \mid \Psi_{n}^{T}\right\rangle}$ being the $\left|\Psi_{n}^{T}\right\rangle$ linear combinations of pairs of approximated eigenstates obtained with SHDMC. Therefore, the squares correspond to the result that one would had obtained using the approximation of Ref. 5 in Eq. (32) for a very good set of functions. Finally, the up triangles mark the result obtained with $Z_{+}(\beta) \simeq \sum_{n} e^{-\beta\left\langle\left|\Psi_{n}^{T}\right| R^{\hat{-1}} \hat{\mathcal{H}} \hat{R} \mid \Psi_{n}^{T}\right\rangle}$, being $\hat{R}$ a random rotation defined in the small subspace.

Figure 2 shows that as the iteration $\ell$ increases, the $Z_{+}(\beta)$ obtained with all methods increases. Note, that the scale of the $y$ axis does not start from zero. The initialization scheme already produces a basis that retains $90 \%$ of the exact value of the truncated partition function. Similar to previous SHDMC methods, the present generalization optimizes the small basis overlap, not their average energy. The trace of $\hat{\mathcal{U}}$ increases indirectly as the small basis approaches to the subspace of the eigenvectors with lowest energy. Note that the SHDMC results are within $\sim$ $1.5 \%$ below the values obtained analytically by projection into the CI data (red rhombi). This difference is due to the remaining errors in the evolution phase which neglects the projection orthogonal to the small subspace. The method used in Ref. 5 applied to approximated pairs of eigenstates (empty squares) gives results only slightly below the SHDMC values, because the energy difference between eigenstates is much smaller that $\beta^{-1}$. However, a random rotation of the basis that spans the same subspace (up triangles) would had produced a significantly worse 


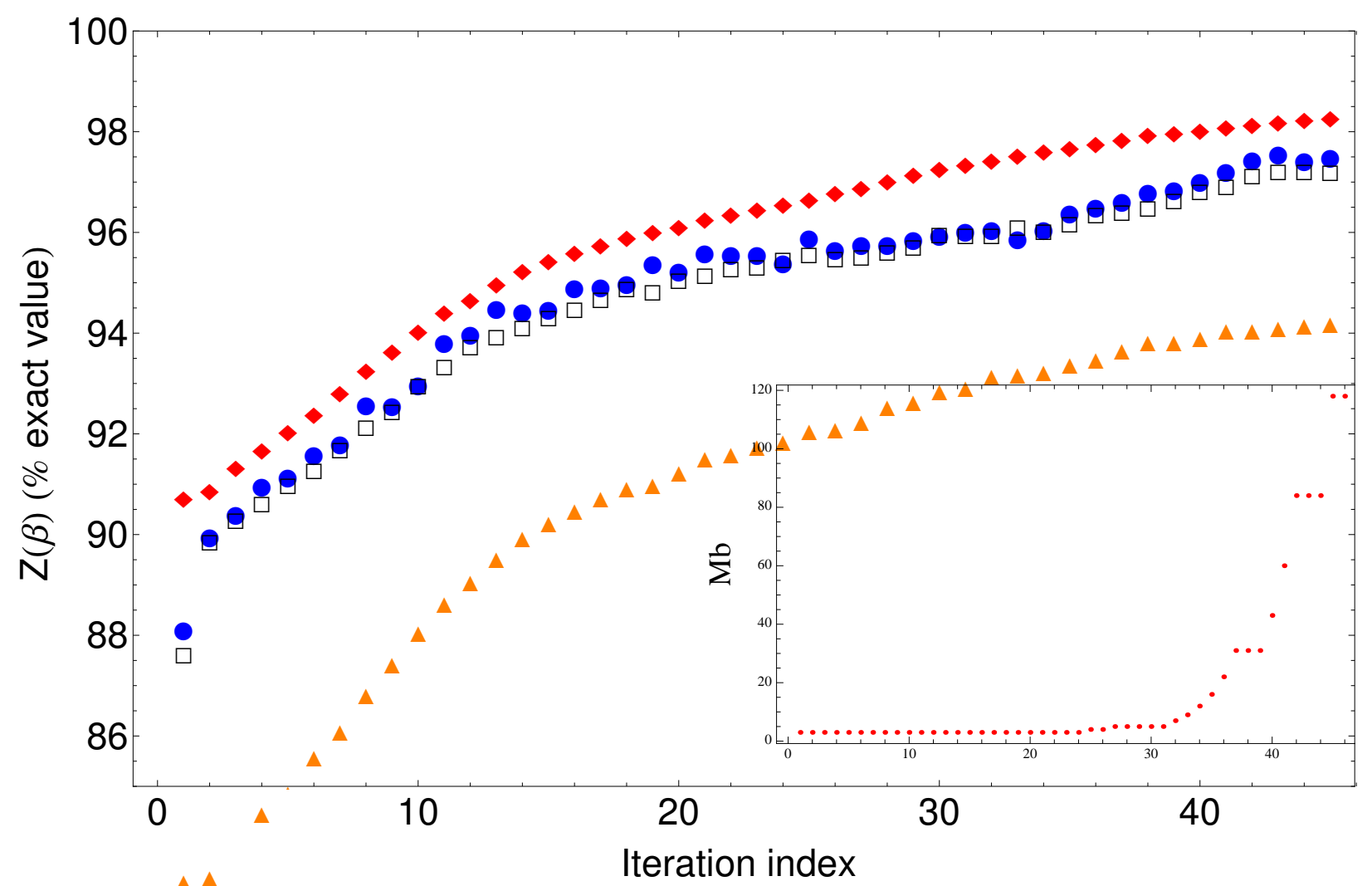

FIG. 2: (Color online) Evolution of the trace of $\hat{\mathcal{U}}=e^{\hat{J}} e^{-\beta \hat{\mathcal{H}}} e^{-\hat{J}}$ in the subspace defined by the small basis $\left\{\left|\chi_{n}^{S, \ell}\right\rangle\right\}$ as a function of the iteration index $\ell$ for $M_{S}=20$ relative to the truncated trace obtained with full CI eigenvalues. Note that the scale starts from $86 \%$ of the full CI value. The blue circles are results obtained with SHDMC using Eq. (30). The red rhombi denote the exact evaluation of $\hat{\mathcal{U}}=e^{\hat{J}} e^{-\beta \hat{\mathcal{H}}} e^{-\hat{J}}$ in the small basis, using full CI data. The empty squares (up triangles) were obtained by evaluating $\left\langle\Psi_{n}^{T}|\hat{\mathcal{H}}| \Psi_{n}^{T},\right\rangle$ for linear combination of eigenstate pairs (random rotation within the small subspace) in the full CI basis while $Z(\beta)$ was obtained using an lower bound approximation. $\frac{5}{5}$ The inset shows the computational cost, the number of sampling subblocks $M_{b}$, as a function of the iteration.

result. This shows that the approach described in Ref. 12 is significantly worse if each element of the basis does not have a large projection on each eigenstate.

Note in the inset of Fig. 2 that the number of sampling blocks remains very low $\left(M_{b}=3\right)$ for the first 25 iterations and starts increasing when the noise becomes dominant around $\ell=32$. If one considers only the first 32 iterations a significant improvement of the partition function is achieved with little computational cost as compared with the calculation of individual eigenstates.

Figure 3 quantifies the convergence of the small basis $\left\{\left|\chi_{n}^{S, \ell}\right\rangle\right\}$ towards the basis defined by the 
eigenstates of the CI $\left\{\left|\Psi_{n}\right\rangle\right\}$. For that purpose we define the logarithm of the residual subspace projection as

$$
L R_{s p}=\ln \left\{1-\left|\operatorname{Det}\left[\left\langle\Psi_{n} \mid \chi_{m}^{S, \ell}\right\rangle\right]\right|^{1 / M_{S}}\right\}
$$

In Eq. (40) Det $\left[\left\langle\Psi_{n} \mid \chi_{m}^{S, \ell}\right\rangle\right]$ is the Determinant of a square matrix of size $M_{S}$ formed by the overlap $\left\langle\Psi_{n} \mid \chi_{m}^{S, \ell}\right\rangle$. The determinant of the matrix is a complex number of modulus 1 in the limit when any eigenstate $\left|\Psi_{n<M_{S}}\right\rangle$ can be written as a linear combination of $\left|\chi_{m}^{S, \ell}\right\rangle$. Any error in any member of the small basis $\left\{\left|\chi_{m}^{S, \ell}\right\rangle\right\}$ reduces the modulus of the determinant by a factor. The exponent $1 / M_{S}$ in Eq. (40) is a standard geometric average. A large negative value in Eq. (40) indicates a very good small basis with a determinant that is approaching 1.

Figure 3 shows the evolution of $L R_{s p}$ given by Eq. (40) as a function of the iteration index $\ell$ for the same system described in Fig. 2. Note that $L R_{s p}$ becomes increasingly negative as a function of $\ell$, which implies a global improvement of the basis approaching to the one described by the eigenstates of the full $\mathrm{CI}$.

We next need to characterize how well an individual eigenstate can be described by the small basis. To measure this we define the logarithm of the residual projection $L R_{n}$ as

$$
L R_{n}=\ln \left[1-\sum_{m=0}^{M_{S}-1}\left|\left\langle\Psi_{n} \mid \chi_{m}^{S, \ell}\right\rangle\right|^{2}\right] .
$$

Note in Eq. (41) that, if the normalized eigenstate can be written as a linear combination of the small basis $\left\{\left|\chi_{m}^{S, \ell}\right\rangle\right\}$, the expression in the brackets should be zero. A large negative number in $L R_{n}$ implies that the eigenstate $\left|\Psi_{n}\right\rangle$ is very well described in the small basis.

Figure 4 describes the evolution of $L R_{n}$ for different eigenstates of the $\mathrm{CI}$ as a function of the iteration index $\ell$.

The blue (red) contribution to the color decreases (increases) as the index $n$ increases. The continuous line follows the ground state. One can clearly observe that the ground state of the CI is already very well described at the initialization stage within the Lanczos-like setup. As the iteration $\ell$ increases, the small basis describes the lowest-energy excitations better while higher excitations require more iterations. Note that for 25 iterations 15 eigenvectors are very well described within a basis of 20 states. The total cost at this point is 300000 individual DMC steps. The calculations of 15 eigenstates with the original SHDMC algorithm for excited states ${ }^{47}$ would had cost at least twice as much. The current algorithm becomes competitive, in addition, if one 


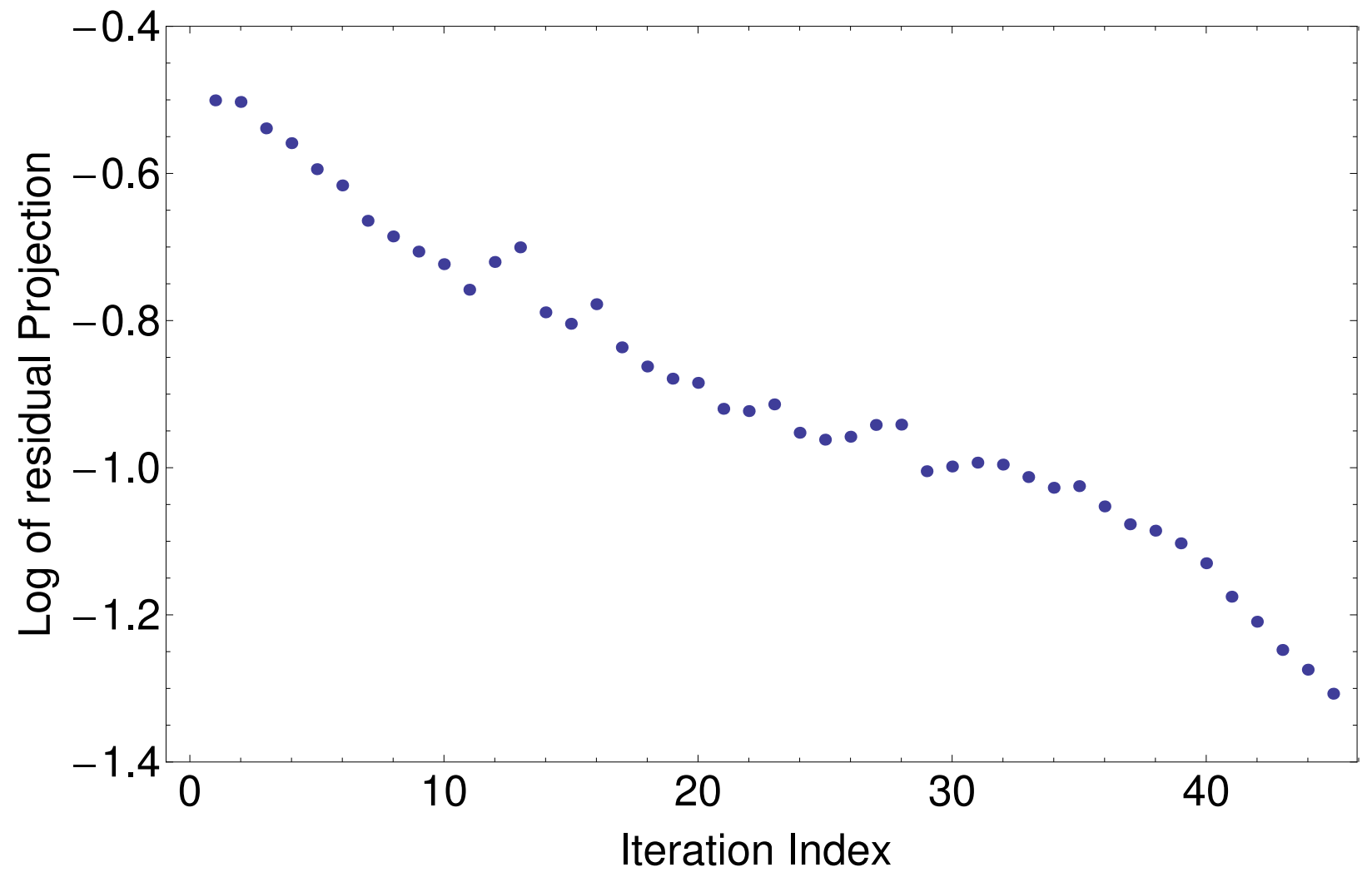

FIG. 3: Logarithm of the residual subspace projection $L R_{s p}$ [see Eq. 40] of the small basis $\left\{\left|\chi_{n}^{S, \ell}\right\rangle\right\}$ into the basis $\left\{\left|\Psi_{n}\right\rangle\right\}$ defined by the eigenstates of the full CI as a function of the iteration index $\ell$ for the same system described in Fig 2, A larger negative number correspond to in improved small basis.

considers that it is parallel, which allows to distribute this cost in multiple tasks $\left(M_{S}\right)$ reducing the time to solution to $2 \%$ as compared with the original SHDMC algorithm for excited states.

Finally, for infinite statistics one could in principle obtain the eigenenergies of $\hat{\mathcal{H}}$ from the eigenvalues of $u_{n}$ as $E_{n} \approx-\ln \left(u_{n}\right) / \beta$. This procedure is known to be inefficient to obtain the eigenenergies which are better described by sampling $\hat{\mathcal{H}}$ as in the CFDMC approach. The off diagonal noise in the matrix elements of $\hat{\mathcal{U}}$ has a perverse effect on the magnitude of the eigenvalues. Therefore, while this method is an efficient one to optimize the basis, it should be combined with other methods to obtain the eigenvalue spectra.

\section{SUMMARY AND DISCUSSIONS}

In this paper we have presented a general framework aimed to calculate thermodynamical properties of many-body system in an importance sampling DMC context..$^{10}$ 


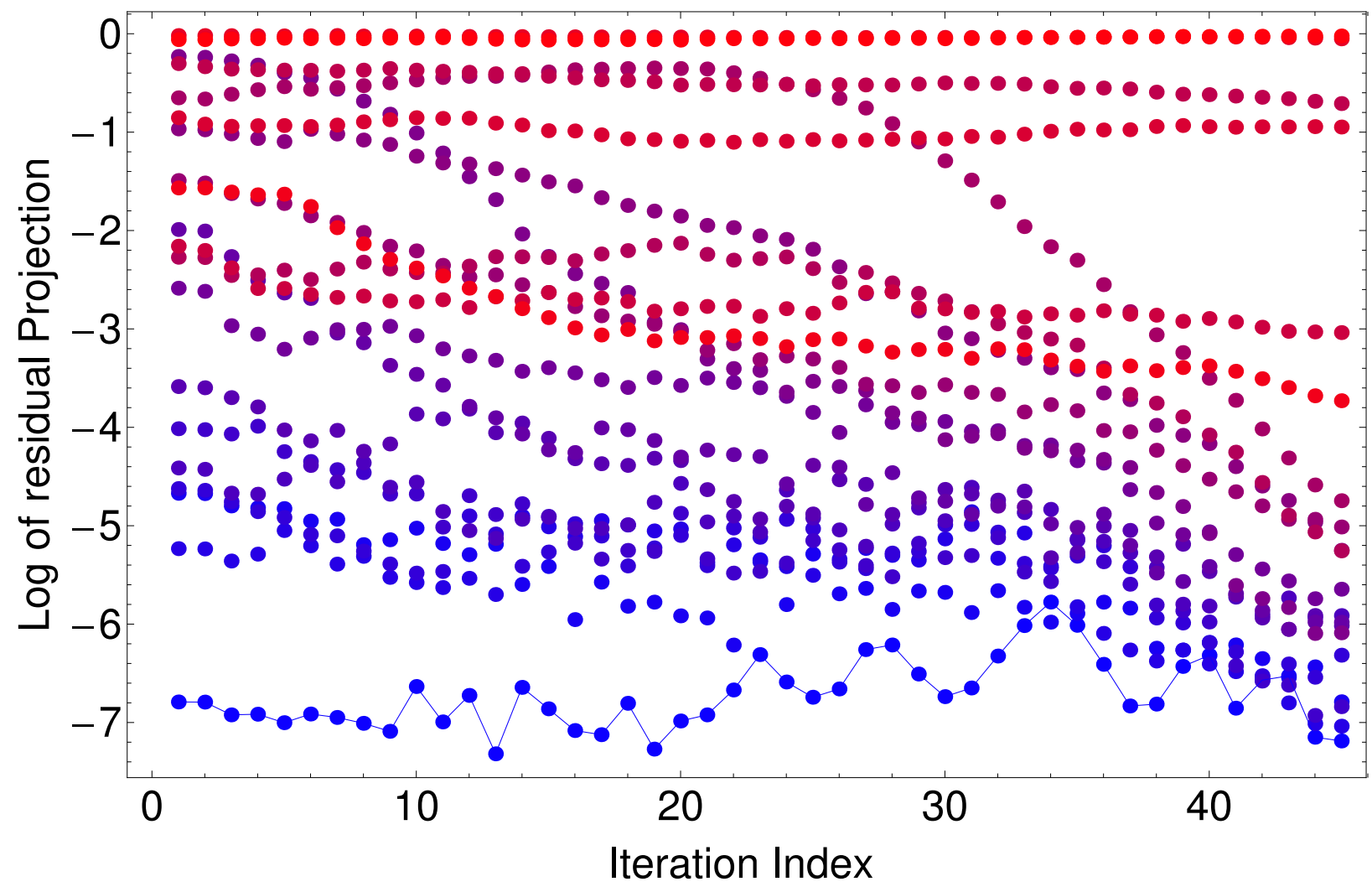

FIG. 4: (Color online) Evolution of the logarithm of residual projection $L R_{n}$ as a function of the iteration index $\ell$. Blue (red) color denotes lower (higher) values of $n$. The continuous line follows the ground state eigenstate.

We showed that a many-body basis can be optimized to describe a small subspace maximizing the overlap with the subspace described by the lowest eigenstates of the Hamiltonian. The Helmholtz free energy obtained within this truncated basis is an upper bound of the exact free energy of a system. The corresponding partition function is a lower bound of the exact partition function.

This generalization of the SHDMC method for finite temperature takes advantage of complex wave functions that do not have nodal pockets. Accordingly, we avoid the appearance of the artificial potentials in the standard fixed-node approximation when the amplitude of the importance sampling guiding function is zero. The antisymmetric properties of the wave-function are enforced by a complex phase. This introduces a complex contribution to the local energy. The complex local energy is handled in the complex weight of the walkers. Going beyond the localtime approximation used in Ref. 42, the evolution of the complex phase factor for $\beta>0$ is now approximately taken into account in the evaluation local energy. The evolution in $\beta$ becomes exact 
as the statistical error is reduced as the sampling increases.

In variance CFDMC, 39 the solution remains an upper bound of the fermionic free energy. While the CFDMC approach uses a bosonic-trial wave function without nodes, SHDMC uses a complex linear combination of anti-symmetric functions without nodal pockets. The walker distribution is prevented to fall into the bosonic ground state solution by the phase factor of the guiding function, which remains antisymmetric, and introduces an effective potential in the local energy. $\stackrel{31,42}{\underline{2}}$

The present approach shares many aspects of the SHDMC method for complex wave functions, but it also incorporates a key advantage of the $\mathrm{CFDMC}^{39}$ approach: several wave functions are optimized at the same time. In systems where many excitations can be approximated by a single amplitude $\Phi(\mathbf{R})$ and a different phase factor $e^{\mathbf{i} \phi_{n}(\mathbf{R})}$, a correlated sampling approach that reweighs the walkers in Eq. (18) as $W_{i} \rightarrow W_{i} \Phi_{n}^{T}(\mathbf{R}) / \Phi(\mathbf{R})$ and changes the phase contribution to the local energy in Eq. (16), would save significant time. If that approximation were used, this generalization of the SHDMC method would look very similar in spirit with CFDMC, the main difference being the use of a complex guiding functions that prevents the exponential growth of the bosonic ground state.

The displacement of each wave function in the small basis during the DMC process is decomposed into a displacement within the subspace already described by the other elements of the small basis plus a contribution orthogonal to the small subspace. The displacement included within the basis is used to improve upon the locality $\frac{57}{2}$ and local-time ${ }^{42}$ approximations. The displacement orthogonal to the small subspace is used to correct the small basis used in the next iteration.

The serial orthogonalization step required in the original SHDMC algorithm for excited states $\frac{42,47}{}$ is avoided with a method that allows the calculation of multiple wave functions in parallel. In addition, the complications of inequivalent nodal pockets of excited states ${ }^{47}$ is avoided using complex trial wave functions without nodes.

The scaling of the cost of an individual iteration of this method is proportional to $M_{S} \times N_{c}$; $M_{S}$ the size of the small basis and $N_{c}$ the number of configurations of the DMC run. The cost of and individual DMC step is dependent of the number of basis functions $N_{b}$ and electrons.

It is well known that as the size of the system increases, the number of basis functions $N_{b}$ required to maintain a fixed error bar for a given eigenstate must increase factorially. But in practice, the error required for evaluation of thermodynamical properties is determined by $\beta^{-1}$ : errors must be much smaller than the temperature of the system. Therefore, as the temperature increases and averages of multiple eigenstates are obtained, the detail required by calculations of the ground 
state energies with chemical accuracy is no longer necessary. The present approach can take advantage of the acceleration of the algorithms used to evaluate large numbers of determinants. $\underline{62.63}$ For a very large $N_{b}$, the cost of these algorithms scales as $N_{e} \times N_{b}$.

The total cost is dependent on the physical system and the goal of the calculation. If the goal is to converge the entire basis or to optimize the Free energy, the bottleneck for convergence is the energy gap $E_{M_{S}+1}-E_{M_{S}}$ which determines the convergence of the basis towards the highest eigenstates considered. Accordingly, in this case, the ideal situation for this method would be a system with $\left(M_{S}\right)$ nearly degenerate eigenstates well-separated from the rest of the spectra within an energy scale of $k_{b} T$. If the goal, instead, is to converge the small basis so as only the lowest $M_{L}$ eigenstates are well described, the convergence of the algorithm is much faster and it is limited by the number of statistical samples and the exponential decay $e^{-\beta\left(E_{M_{S}+1}-E_{M_{L}}\right)}$. The cost is reduced as compared with the calculation of eigenstates if one accepts an error in the higher excitations. If one wishes to retain the physics of higher eigenstates in the basis, it is computationally more efficient to increase $M_{S}$ (which increases the cost linearly), instead of improving the basis for the higher excitations which increases the cost exponentially.

Comparisons of the method with full CI calculations show that SHDMC can be used to optimize many-body basis sets to maximize the overlap with the lowest energy excitations of the Hamiltonian. Each eigenenergy obtained with this method has lower quality than those obtained with alternative approaches such as LMMC or the standard SHDMC for excited states. However, this method could be a useful tool to optimize the basis, minimizing the size of the matrices used in LMMC and thus reducing the effects of numerical noise in LMMC.

\section{Acknowledgments}

The authors would like to thank J. Krogel and P. R. C. Kent for a critical reading of the manuscript and discussions. This work has been supported by the grant ERKCS92 Materials Science and Engineering division of Basic Energy Sciences, Department of Energy.

\section{Appendix A: Going beyond the locality and local-time approximations}

Approximate coefficients for the Slater determinant expansion of the eigenstates of $\hat{\mathcal{H}}$ and $e^{-\beta \hat{\mathcal{H}}}$ can be obtained from the eigenstates of $\hat{\mathcal{U}}=e^{\hat{J}} e^{-\beta \hat{\mathcal{H}}} e^{-\hat{J}}$ in the $\left\{\chi_{n}^{S}\right\}$ basis [see Eq. (28)]. But, $\hat{\mathcal{U}}$ is 
not hermitian, since $\hat{\mathcal{U}}^{\dagger}=e^{2 \hat{J}} \hat{\mathcal{U}} e^{-2 \hat{J}}$. Nevertheless, as long as the Jastrow factor operator $e^{-\hat{J}}$ has an inverse, $\hat{\mathcal{U}}$ has a set of right eigenvectors $\left|\chi_{i}^{U}\right\rangle=e^{\hat{J}}\left|\Psi_{i}\right\rangle$ and left eigenvectors $\left\langle\chi_{i}^{U}\right|=\left\langle\Psi_{i}\right| e^{-\hat{J}}$. Since $\hat{\mathcal{H}}$ is Hermitian, its eigenstates $\left|\Psi_{i}\right\rangle$ are orthogonal, which implies that $\left\langle\chi_{i}^{U} \mid \chi_{j}^{U}\right\rangle=\delta_{i, j}$. Within statistical error bars, in the small subspace, the matrix elements of $\hat{\mathcal{U}}$ obtained with the basis $\left|\chi_{n}^{S, \ell}\right\rangle$ of the iteration $\ell$ are given by Eq. (28). In the first iteration the matrix elements of $\hat{\mathcal{U}}$ can be obtained directly from the Lanczos-like procedure.

Within the small subspace, $\hat{\mathcal{U}}$ can be written as

$$
\hat{\mathcal{U}}=\sum_{i} u_{i}\left|\chi_{i}^{U}\right\rangle\left\langle\chi_{i}^{U}\right|
$$

Since the $u_{i}$ are also the eigenvalues of $e^{-\beta \hat{\mathcal{H}}}$ their dependence with $\beta$ is exponential. Thus for an arbitrary $\beta^{\prime}$ the eigenvalue will be $u_{i}^{\beta^{\prime} / \beta}$.

Provided that the difference $\left|\Delta \tilde{\chi}_{n}^{S, \ell+1}\right\rangle=\left|\chi_{n}^{S, \ell+1}\right\rangle-\left|\chi_{n}^{S, \ell}\right\rangle$ is small [which is always valid for $\beta \rightarrow 0$ see Eq. (38)], the dependence in $\beta$ of $\left|\chi_{n}^{S, \ell+1}(\beta)\right\rangle$ can be approximated as follows: Let's first define the operator $\hat{R}=\sum_{n=0}^{M_{S}-1}\left|\chi_{n}^{S, \ell}\right\rangle\left\langle\chi_{n}^{S, \ell+1}\right|$ and its inverse within the small subspace $\left.\hat{R}^{\dagger}=\sum_{n=0}^{M_{S}-1} \| \chi_{n}^{S, \ell+1}\right\rangle\left\langle\chi_{n}^{S, \ell}\right|$.

Accordingly, the dependence in $\beta^{\prime}$ of the new basis can be approximated as

$$
\left|\chi_{n}^{S, \ell+1}\left(\beta^{\prime}\right)\right\rangle=\hat{R}^{\dagger} \hat{\mathcal{U}}^{\beta^{\prime} / \beta} \hat{R}\left|\chi_{n}^{S, \ell+1}\right\rangle
$$

with $\hat{\mathcal{U}}^{\beta^{\prime} / \beta}$ given by Eq. (A1) replacing $u_{i}$ by $u_{i}^{\beta^{\prime} / \beta}$.

\section{Appendix B: Working with eigenstates of $\hat{\mathcal{U}}$}

While in some systems eigenstates of $\hat{\mathcal{H}}$ are always real (e.g. confined systems with time reversal symmetry), in many cases the wave function of the eigenstates is known to be complex, without nodal pockets. In those cases Eq. (13) is valid and no walker needs to be killed or rejected because the eigenstate wave function does not have a nodal surface. ${ }^{42}$ In these cases it might be advantageous to propagate single eigenstates of $\hat{R}^{\dagger} \hat{\mathcal{U}}^{\frac{j}{k}} \hat{R}$, since the variance of the weights is minimized and lower (larger) $T(\beta)$ can be reached with less statistical data. An additional advantage of working with functions that are closer to the eigenstates is that the locality and localtime approximations can be used.

Since any $\left|\chi_{n}^{S}\right\rangle=\sum_{i=0}^{M_{S}-1}\left\langle\chi_{i}^{X} \mid \chi_{n}^{S}\right\rangle\left|\chi_{i}^{X}\right\rangle$ can be written as a linear combination of $\left|\chi_{i}^{X}\right\rangle$ and vise versa, when eigenstates are complex one can use as trial wave function $e^{\hat{J}}\left|\chi_{i}^{U}\right\rangle$ in the SHDMC 
propagation and sampling. Then the propagation of $\left|\chi_{n}^{S}\right\rangle$ can be written as a linear combination of the propagation of the eigenstates of $\hat{\mathcal{U}}$. as:

$$
\begin{aligned}
\left|\delta \chi_{n}^{S}\right\rangle & =\sum_{i=0}^{M_{S}}\left\{\left\langle\chi_{i}^{X} \mid \chi_{n}^{S}\right\rangle e^{-\beta\left(E_{i}^{X}-E_{n}^{S}\right)}\left[\left|\chi_{i}^{X}\right\rangle+\left|\delta \chi_{i}^{X}\right\rangle\right]\right\}-\left|\chi_{n}^{S}\right\rangle \text { and } \\
\left|\delta \tilde{\chi}_{n}^{S}\right\rangle & =\sum_{i=0}^{M_{S}}\left\{\left\langle\chi_{i}^{X} \mid \chi_{n}^{S}\right\rangle e^{-\beta\left(E_{i}^{X}-E_{n}^{S}\right)}\left[\left|\chi_{i}^{X}\right\rangle+\left|\delta \tilde{\chi}_{i}^{X}\right\rangle\right]\right\}-\left|\chi_{n}^{S}\right\rangle
\end{aligned}
$$

$E_{n}^{S}$ results from the condition $\left\langle\chi_{n}^{S} \mid \delta \chi_{n}^{S}\right\rangle=0$ and it is given by

$$
e^{-\beta E_{n}^{S}}=\sum_{i}\left[\left|\left\langle\chi_{i}^{X} \mid \chi_{n}^{S}\right\rangle\right|^{2}+\left\langle\chi_{i}^{X} \mid \chi_{n}^{S}\right\rangle\left\langle\chi_{n}^{S} \mid \delta \chi_{i}^{X}\right\rangle\right] e^{-\beta E_{i}^{X}}
$$

replacing $X$ by $U$ in Eqs. ( 1 11) and (B2). The brackets $\left\langle\chi_{i}^{U} \mid \chi_{n}^{S}\right\rangle$ are obtained by diagonalizing $\hat{\mathcal{U}}$ : the coefficient $n$ of the left eigenvectors $i$ in the small basis $\left\{\left\langle\chi_{n}^{S}\right|\right\}$.

The disadvantages is that complex eigenstates appear only in certain Hamiltonians or boundary conditions. Albeit without nodes, they might have large variation in probability density, in particular for small magnetic fields or twist boundary conditions close to high symmetry points. Large variations in the probability density hinder correlating sampling.

\section{Appendix C: Working with eigenstates pairs}

It is well known that in many physical systems the energy spacing between eigenstates decreases as the size of the system increases. It is also known that the variance of the local energy, which is related to the statistical error in the energy, increases as the size of the system increases. ${ }^{64}$ Accordingly as the size of the system increases, it becomes more difficult to obtain eigenstates. As the size of the system increases the error in the variance introduced by a linear combination of eigenstates in the Slater part of the wave function becomes smaller than the variance introduced by short range correlations. These short range correlations cannot be accounted by the Slater part, even with a very large basis $\{|n\rangle\}$, or with simple Jastrow factors. Accordingly, in large systems little is lost by using a linear combination of eigenstates, since their contribution to the variance is proportional to the energy separation that decreases as the system become larger. In contrast, much is gained avoiding the divergences at the nodes by using complex linear combinations of eigenstates, in particular, to obtain average of thermodynamical properties. However, to propagate for larger $\beta$ with a branching algorithm, it will be necessary to minimize the variance of the local energy. 
If the eigenstates are real, one must use a linear combination of eigenstates. The minimum variance will be reached by constructing the Slater part of the guiding wave function with linear combinations of eigenstates with consecutive eigenvalues of $u_{i}$. In this work we use

$$
\begin{aligned}
\left|\chi_{2 j-1}^{V}\right\rangle & =\frac{1}{\sqrt{2}}\left(\left|\chi_{2 j-1}^{U}\right\rangle+e^{\mathbf{i} \phi}\left|\chi_{2 j}^{U}\right\rangle\right) \\
\left|\chi_{2 j}^{V}\right\rangle & =\frac{1}{\sqrt{2}}\left(\left|\chi_{2 j-1}^{U}\right\rangle-e^{\mathbf{i} \phi}\left|\chi_{2 j}^{U}\right\rangle\right),
\end{aligned}
$$

where $e^{\mathbf{i} \phi}$ is a complex phase which can be adjusted to minimize the variance of the amplitude of the complex wave function $\Phi_{n}^{T}(\mathbf{R})$. The conjugate vectors $\left\langle\chi_{2 j-1}^{V}\right|$ are constructed using complex conjugate coefficients and the left eigenvectors of $\hat{\mathcal{U}}$ in the small basis $\left\{\left\langle\chi_{n}^{S}\right|\right\}$.

Their evolution in imaginary time is given by

$$
\begin{aligned}
\left|\chi_{2 j-1}^{V}\left(\beta^{\prime}\right)\right\rangle & =\frac{1}{\sqrt{2}}\left[\left(u_{2 j-1}\right)^{\frac{\beta^{\prime}}{\beta}}\left|\chi_{2 j-1}^{U}\right\rangle+e^{\mathbf{i} \phi}\left(u_{2 j}\right)^{\frac{\beta^{\prime}}{\beta}}\left|\chi_{2 j}^{U}\right\rangle\right] \\
\left|\chi_{2 j}^{V}\left(\beta^{\prime}\right)\right\rangle & =\frac{1}{\sqrt{2}}\left[\left(u_{2 j-1}\right)^{\frac{\beta^{\prime}}{\beta}}\left|\chi_{2 j-1}^{U}\right\rangle-e^{\mathbf{i} \phi}\left(u_{2 j}\right)^{\frac{\beta^{\prime}}{\beta}}\left|\chi_{2 j}^{U}\right\rangle\right]
\end{aligned}
$$

1 C. Kittel Quantum Theory of Solids (John Wiley \& Sons, New York, 1987).

2 E. Runge, E. K. U. Gross, Phys. Rev. Lett. 52, 997-1000 (1984).

3 G. Onida, L. Reining, A. Rubio, Rev. Mod. Phys. 74, 601-659 (2002).

4 C. Filippi, M. Zaccheddu and F. Buda. Chem. Theory Comput.5, 20742087 (2009).

5 G. Mazzola, A. Zen, and S. Sorella J. Chem. Phys. 137, 134112 (2012).

6 J.C. Grossman and L. Mitas, Phys. Rev. Lett. 79, 4353-4356, (1997).

7 S. Saccani, C. Filippi, S. Moroni http://arxiv.org/abs/1211.5462 (2013).

8 B. G. Levine, T. J. Martinez, in Annual Review of Physical Chemistry 58, 613-634 (2007).

9 M. A. Morales, C. Pierleoni, D. M. Ceperley, Phys. Rev. E. 81, 021202 (2010).

10 D. M. Ceperley and B. J. Alder, Phys. Rev. Lett. 45, 566 (1980).

11 P. Hohenberg and W. Kohn, Phys. Rev. 136, B864 (1964).

12 W. Kohn and L. J. Sham, Phys. Rev. 140, A1133 (1965).

13 N. D. Mermin, Phys. Rev. 137, A1441 (1965).

14 W. Kohn and P. Vashishta. in Theory of the inhomogeneous electron gas (S. Lundqvist and N. H. March eds.) New York: Plenum, pp. 79-147 (1983). 
M. Weinert and J. W. Davenport Phys. Rev. B 45, 13709 (1992).

16 J. Staunton, B. L. Gyorffy, A. J. Pindor, et al. J. of Mag. and Mag. Mat. 45, 15-22 (1984).

17 B. L. Gyorffy, A. J. Pindor, J Staunton, et al. J. of Phys. F 15, 1337-1386 (1985).

18 J. Staunton, B. L. Gyorffy, A. J. Pindor, et al. J. of Phys. F 15, 1387-1404 (1985).

19 Y. Wang, G. M. Stocks, W. A. Shelton, D. M. C. Nicholson, Z. Szotek and W. M. Temmerman, Phys. Rev. Lett. 75, 2867 (1995).

20 D. P. Landau, S.-H. Tsai, and M. Exler, American Journal of Physics, 72, 12941302, (2004).

21 M. Eisenbach, C.-G. Zhou, D. M. Nicholson, G. Brown, J. Larkin, and T. C. Schulthess, SC '09: Proceedings of the Conference on High Performance Computing Networking, Storage and Analysis, ACM (2009).

22 J. Yin, M. Eisenbach, D. M. Nicholson, A. Rusanu, Phys. Rev. B 86, 214423 (2012).

23 D. M. Ceperley, Rev. of Mod. Phys. 67, 279-355 (1995).

24 W. R. Magro, D. M. Ceperley, C, Pierleoni, and B. Bernu, Phys. Rev. Lett. 76,1240-1243 (1996).

25 B. Militzer, D. M. Ceperley, Phys. Rev. Lett. 85, 1890-1893 (2000).

26 K.P. Driver, B. Militzer, B. , Phys. Rev. Lett. 108, 15502 (2012).

27 W. Purwanto, S. Zhang, and H. Krakauer, J. Chem. Phys. 130, 094107 (2009).

28 G. H. Booth, A. Grneis, G. Kresse, and A. Alavi Nature 493, 365370 (2012).

29 W. M. C. Foulkes, L. Mitas, R. J. Needs, and G. Rajagopal, Rev. Mod. Phys. 73, 33 (2001).

30 B. M. Austin, D. Y. Zubarev,W. A. Lester, Chem. Rev. 112, 263-288 (2012).

31 G. Ortiz, D. M. Ceperley, and R. M. Martin, Phys Rev. Lett. 71, 2777 (1993).

32 G. Ortiz, and D. M. Ceperley Phys. Rev. Lett. 75, 4642 (1995).

33 M. D. Jones, G. Ortiz, and D. M. Ceperley, Phys. Rev. E, 55, 6202, (1997).

34 A. D. Güçlü and C. J. Umrigar, Phys. Rev. B, 72, 045309 (2005); A. D. Güçlü, G. S. Jeon, C. J. Umrigar and J. K. Jain, Phys. Rev. B 72, 205327 (2005); G. S. Jeon, A. D. Güçlü, C. J. Umrigar, and J. K. Jain, Phys. Rev. B 72, 245312, (2005).

35 C. J. Umrigar, J. Toulouse, C. Filippi, S. Sorella, and R. G. Hennig, Phys. Rev. Lett. 98, 110201 (2007).

36 J. Toulouse and C. J. Umrigar, J. Chem. Phys. 128, 174101 (2008).

37 F. R. Petruzielo, Julien Toulouse, C. J. Umrigar, J. of Chem. Phys. 136, 124116 (2012).

38 P. Lòpez-Rios, A. Ma, N. D. Drummond, M. D. Towler, and R. J. Needs, Phys. Rev. E 74, 066701 (2006).

39 D. M. Ceperley and B. Bernu, J. Chem. Phys. 89, 6316 (1988); B. Bernu, D. M. Ceperley, and W. A. 
Lester, Jr., J. Chem. Phys. 93, 552 (1990).

40 F. A. Reboredo, R. Q. Hood, and P. R. C. Kent, Phys. Rev. B 79, 195117 (2009).

41 M. Bajdich, M. L. Tiago, R. Q. Hood, P. R. C. Kent, and F. A. Reboredo, Phys. Rev. Lett. 104, 193001 (2010).

42 F. A. Reboredo J. Chem. Phys. 136, 204101 (2012).

43 J. B. Anderson, Int. J. Quantum Chem. 15, 109 (1979).

44 D. Bressanini Phys. Rev. B 86, 115120 (2012) and references there in.

45 D. M. Ceperley, J. Stat. Phys. 63, 1237 (1991).

46 K. M. Rasch and L. Mitas, Chem. Phys. Lett. 528, 59 (2012).

47 F. A. Reboredo, Phys. Rev. B 80, 125110 (2009).

48 L. E. Reichl A modern Course in Statistical Physics (John Wiley \& Sons, INC., New York,1997)

49 T. Kato, Commun. Pure Appl. Math. 10, 151 (1957).

50 M. Bajdich,* L. Mitas, and L. K. Wagner and K. E. Schmidt, Phys. Rev. B 77, 115112 (2008).

51 J. Toulouse, M. Caffarel, P. Reinhardt, P.E. Hoggan, C. J. Umrigar, In Advances in theory of quantum systems in chemistry and physics Book Series: Progress in Theoretical Chemistry and Physics 22, 343351 (2012).

52 The theory can be extended to a back-flow operator ${ }^{38}$ as long at it has an inverse.

53 Since we used guiding functions without nodal pockets, the complications of inequivalent nodal pockets ${ }^{47}$ are also avoided.

54 The complex logarithm is a multivalued function. See Donald Sarason, "Complex function theory", 2nd ed., Amer. Math. Society, 2007, Section IV.9. Also en.wikipedia.org/wiki/Complex_logarithm.

55 B. L. Hammond, W. A. Lester, Jr., and P. J. Reynolds, Monte Carlo Methods in Ab Initio Quantum Chemistry (World Scientific, Singapore-New Jersey-London-Hong Kong, 1994).

56 D. Meyer, S. Boblest, G. Wunner, Phys. Rev. A 87, 032515 (2013).

57 L. Mitas, E.L. Shirley and D.M. Ceperley J. Chem. Phys. 95, 3467 (1991).

58 The fixed-phase approximation ${ }^{31}$, which corresponds to neglecting the term inside the \{\} in Eq. (12), is never used in this work.

59 C. J. Umrigar, M. P. Nightingale, and K. J. Runge, J. Chem. Phys. 99, 2865 (1993).

60 F. A. Reboredo and P. R. C. Kent, Phys. Rev. B 77, 245110 (2008).

61 Seiichiro Ten-no, Osamu Hino Int. J. Mol. Sci. 3, 459-474 (2002).

62 P. K. V. V. Nukala and P. R. C. Kent, J. Chem. Phys. 130, 204105 (2009). 
63 Clark, B.K., Morales, M.A., McMinis, J., Kim, and G. E. Scuseria, J. of Chem. Phys. 135, 244105 (2011).

64 N. Nemec, Phys. Rev. B 81, 035119 (2010). 